UCID- 21574 DRAFT Rev. 1

\author{
Compendium of Compuzer Codes for the Researcher \\ in Magnetic Fusion Energy
}

Edited by Gary D. Porter

March 10, 1989

This is an informal report intended primarily for internal or limited external distri'ution. The opinions and conclusions stated are .ose of the author and may or may not be those of the Laboratory.

Work performed under the auspices of the L.S. Degartment of Energy by the Lawrence Livermore National Laboratory under Contract W-7405-Eng-48. 


\section{DISCLAIMER}

This document was prepared at an account of work apossored by an agency of the United States Government. Neither itie Iniled States Coverament nor the Universily of Californit wor any of their eaplayeet, anaket any

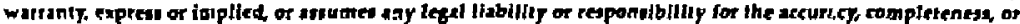

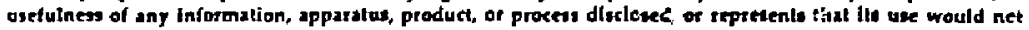

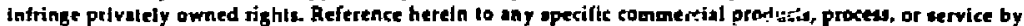
irde name, trademark, mantacturet, or otherwise, does not necess rily conotitute or imply its endortemeni. fecom mendation, of fovoring by the United States Covemment of the Unlvernily of California. The viewa and opinions of authors expressed herein do not retetwarily atale or reflet those of the United Sitates Governement or the Univenity of California, and thall not be used for advertising or product endotsement purposes.

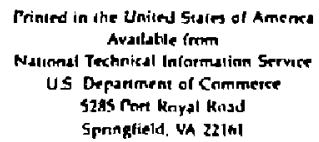

\section{Pupercopy Prices}

$\mathrm{AO2}$

A0.3

AOS

A05

A06

A07

A08

A09
$001-050$

$051-100$

$101-200$

$201-300$

$301-400$

$401-500$

$501-600$

601 


\title{
Compendium of Computer Codes For the Researcher in Magnetic Fusion Energy
}

\author{
G. D. Porter \\ Lawrence Livermore National Laboratory. \\ University of C'alifornia, \\ Livermore, California 94550
}

\begin{abstract}
This is a compendium of computer codes, which are available to the fusion researcher. It is intended to be a document that permits a quick evaluation of the tools available to the experimenter who wants to both analyze his data, and compare the results of his analysis with the predictions of available theories. This document will be updated frequently to maintain its usefulness. I would appreciate receiving further information about codes not included here from anyone who has used them. The information required includes a brief description of the code (including any special features), a bibliography of the documentation available for the code and/or the underlying physics, a list of people to contact for help in ruming the code, instructions on how to access the code, and a description of the output from the code. Wherever possible: the code contacts should include people from each of the fusion facilities so that the novice can talk to someone "down the hall" when he first tries to use a code. I would also appreciate any comments abcut possible additions and improvements ir the index. I encourage any additional criticism of this document.
\end{abstract}

\section{DISCLAIMER}

This repart was prepared as an aceount of work sponsored by an agency of the United States Government. Neither the United States Governgrent nor any agency thereof, nor any of their employees, makes any warranty, express or implied, or assumes any legal liability or responsibility for the accuracy, complet:ness, or uselulness of any information, apparatus, product, or process disclosed, or represents that its use would not infringe privately owned rights. Rererence herein to any specific commercial product, process, or service by trade name, Irademark, manufacturee, or otherwise does not necessarily constitule or imply its endersement. recommendation, or favoring by the United States Government of any agency the cof. The riews and opintons of authors expressed hercin de not necessarily state or refte:t those of the United States Government or any agency thereof.

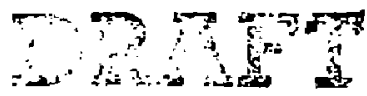


1 Introduction 6

2 General Utility Codes 6

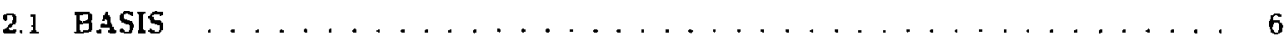

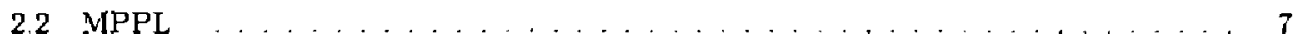

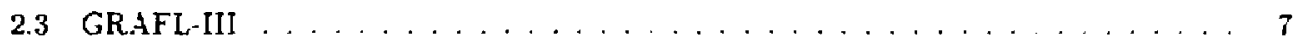

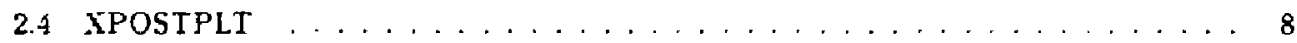

3 General Physics Codes $\quad 8$

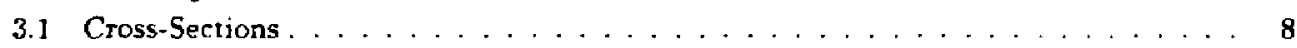

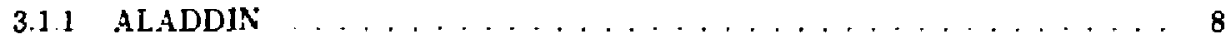

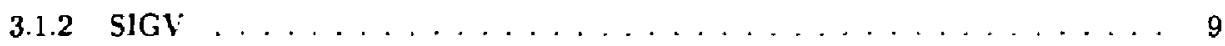

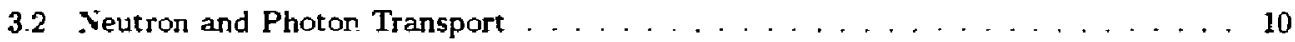

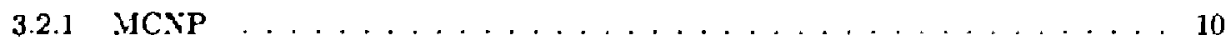

3.3 Vacuum Field Calculation . . . . . . . . . . . . . . . . . 11

3.3 .1 EFFI . . . . . . . . . . . . . . . . . . . . 11

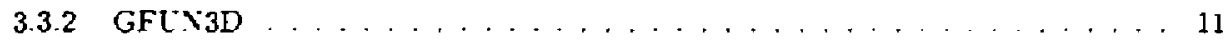

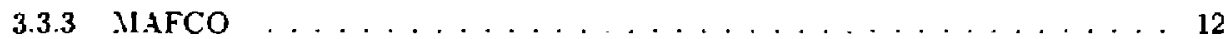

4 Data Analysis Codes $\quad 12$

4.1 General Data Analysis . . . . . . . . . . . . . . . . . . . . . . . . . . . . . . . . . . . . . . . . .

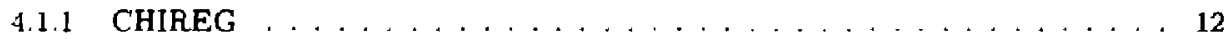

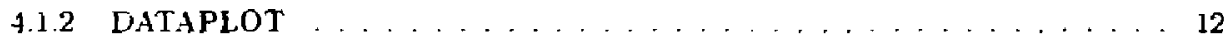

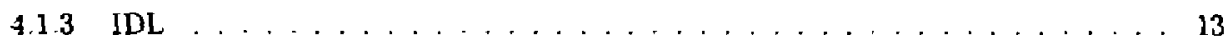

4.1 .4 LOCLS . . . . . . . . . . . . . . . . . . . 14

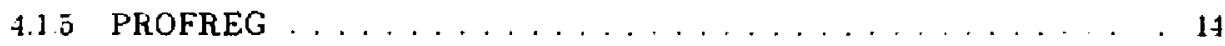

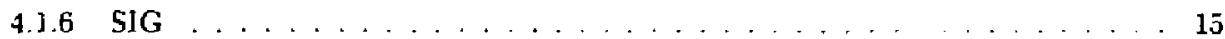

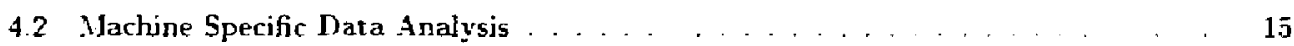

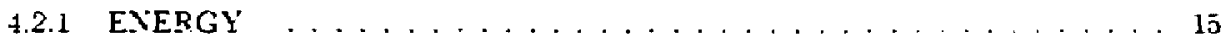

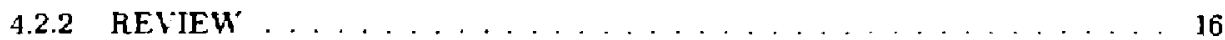

5 Design Codes $\quad 16$

5.1 Tokamaks . . . . . . . . . . . . . . . . . . . . . . . . . . . 16

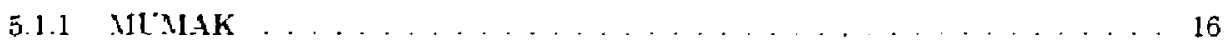

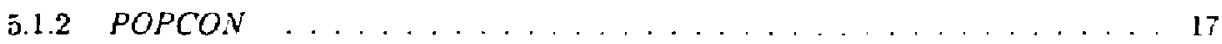

5.1 .3 TADDPACK $\ldots \ldots \ldots \ldots \ldots \ldots$

5.1 .4 TETRA $\ldots \ldots \ldots \ldots \ldots \ldots$

6 MHD Equilibrium Codes $\quad 18$

6.1 Open Geometry . . . . . . . . . . . . . . . . . . . . . . . . . . . . 18

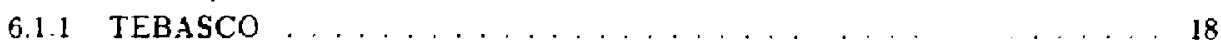

6.1 .2 LTE . . . . . . . . . . . . . . . . . . . . . . . . . . . . . . . . . .

62 Toroidal Geometry . . . . . . . . . . . . . . . . . . . . . . 18 


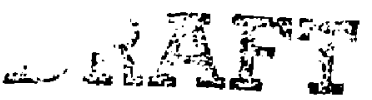

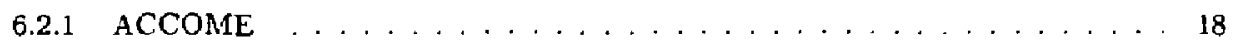

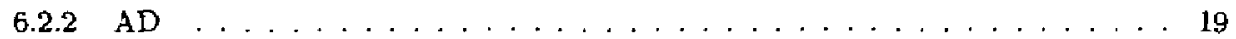

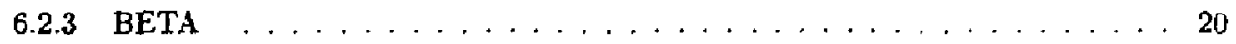

6.2 .4 EFIT . . . . . . . . . . . . . . . . . . . . . 20

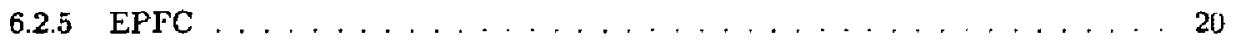

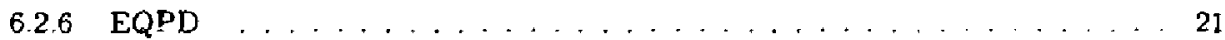

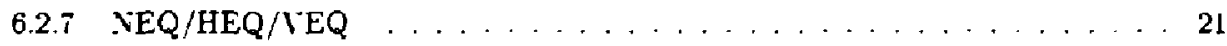

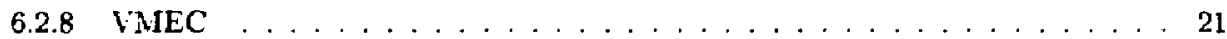

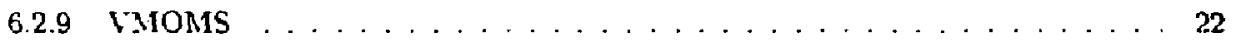

7 Particle Following Codes 23

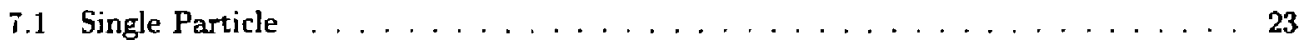

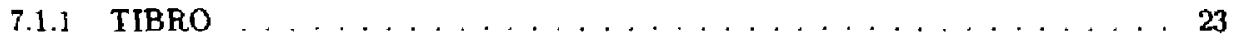

T.2 Monte-Carlo. . . . . . . . . . . . . . . . . . . . . . . . 23

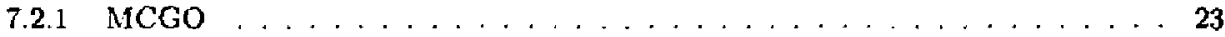

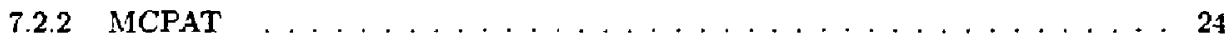

7.3 Particle-in-cell Simulation codes with self-consistent fields . . . . . . . . . 25

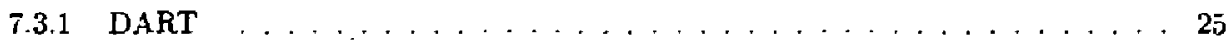

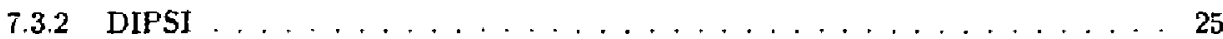

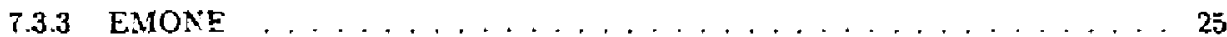

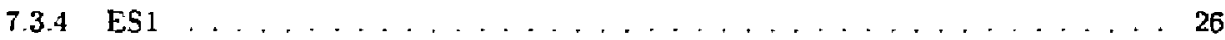

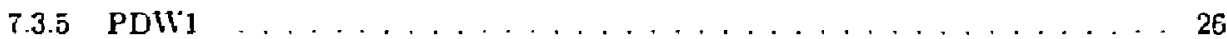

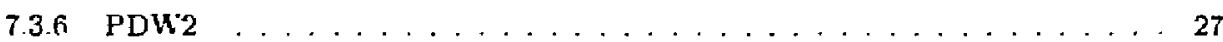

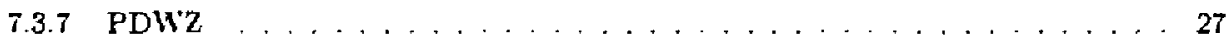

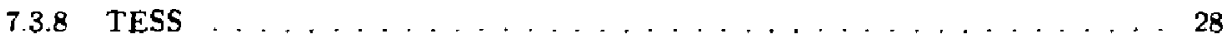

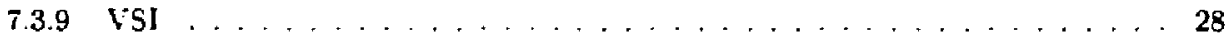

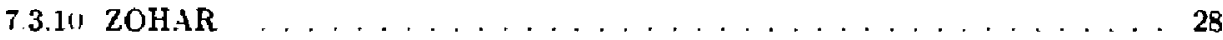

8 Plasma Transport Codes $\quad 29$

8.1 Cross-Field Transport . . . . . . . . . . . . . . . . . . . . . . 29

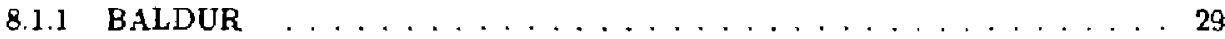

8.1 .2 BETA MC (Monte Carlo) . . . . . . . . . . . . . . 29

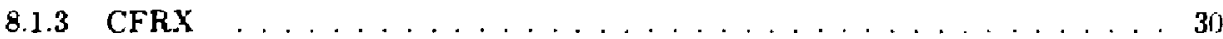

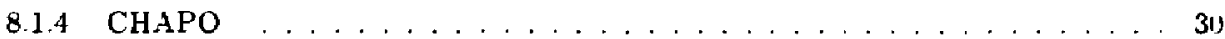

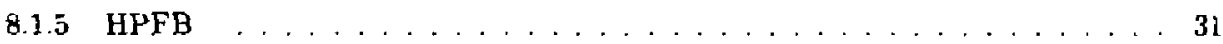

8.1 .6 IHTMDC . . . . . . . . . . . . . . . . . . . . . . .

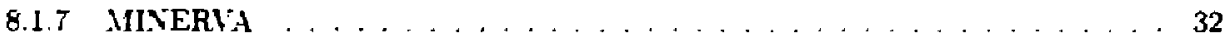

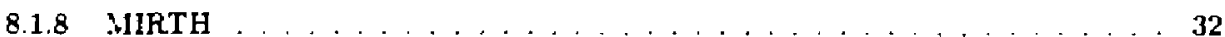

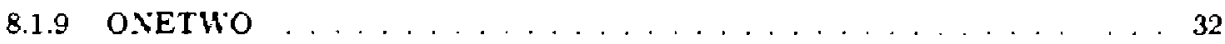

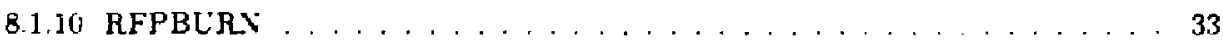

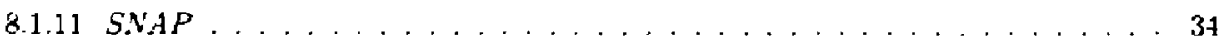

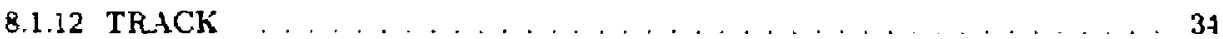

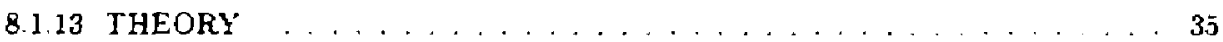




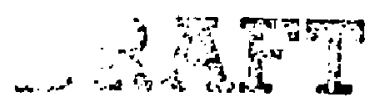

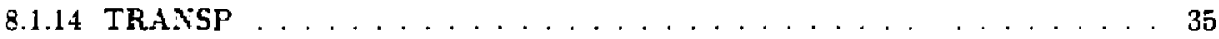

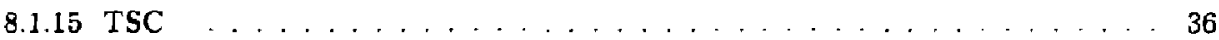

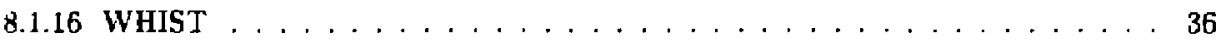

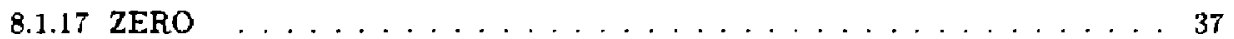

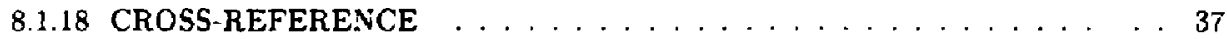

8.2 2-D Traisport . . . . . . . . . . . . . . . . . . . . . 38

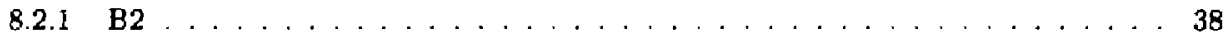

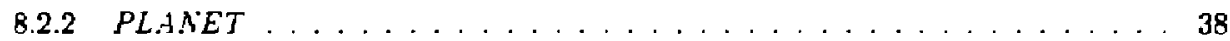

8.3 Parallel Trarsport $\ldots \ldots \ldots \ldots \ldots \ldots \ldots \ldots$

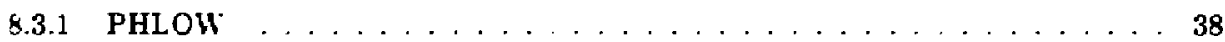

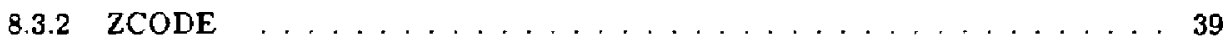

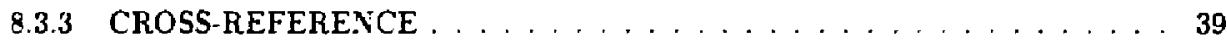

8.4 Impurity Transport . . . . . . . . . . . . . . . . . . . . . . . 40

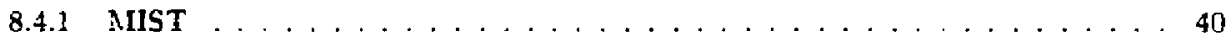

9 Sources and Sinks $\quad \mathbf{4 1}$

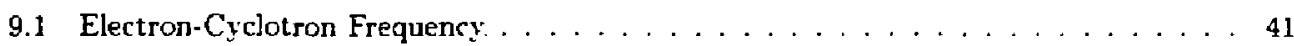

$9.1 .1 \mathrm{TORCH} \ldots \ldots \ldots \ldots \ldots \ldots \ldots \ldots$

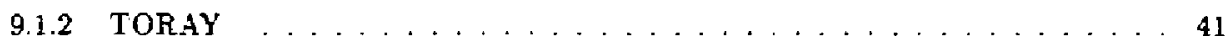

9.2 lon-Cyclotron Frequency $\ldots \ldots \ldots \ldots \ldots \ldots$

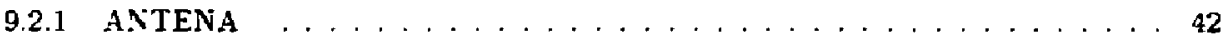

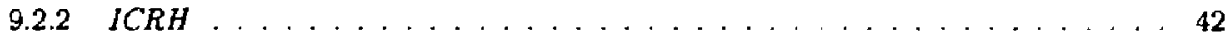

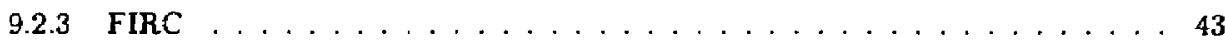

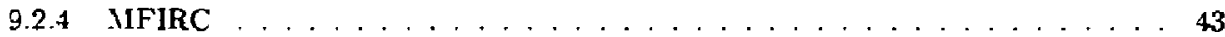

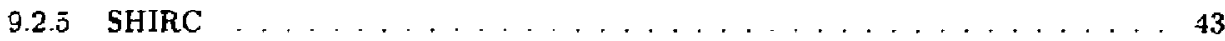

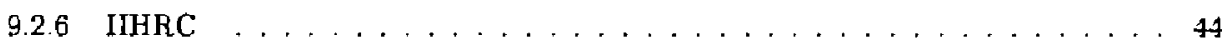

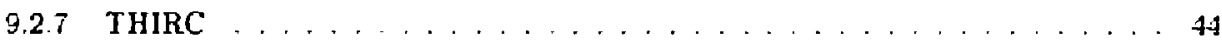

9.2 .8 G.ARFIELD $\ldots \ldots \ldots \ldots \ldots \ldots \ldots \ldots \ldots \ldots \ldots$

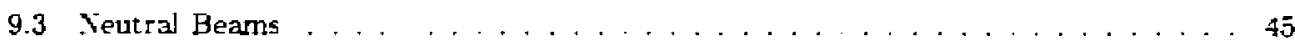

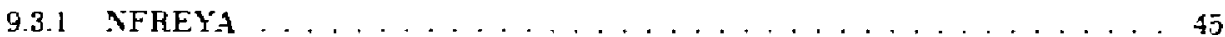

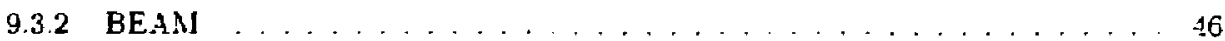

9.4 Veutral Partjcle Transport . . . . . . . . . . . . . . . . . . . . 46

9.4 DEGAS . . . . . . . . . . . . . . . . . . . 46

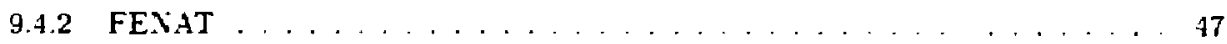

9.4 PELLET . . . . . . . . . . . . . . . . 47

9.5 Impurity Radiation Codes . . . . . . . . . . . . . . . . . . . . . . . . . . 48

9.6 Cyclatron Emission Codes . . . . . . . . . . . . . . . . . . . . . . . . . 48

9.6 .1 CYTRAX . . . . . . . . . . . . . . . . . . . 48

9.6 .2 HORACE $\ldots \ldots \ldots \ldots \ldots \ldots \ldots \ldots$

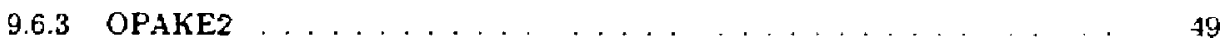

9.7 Lower Hybrid . . . . . . . . . . . . . . . . . . . . . . . . . . . . . . . . . . . . . . . . . . 4.49

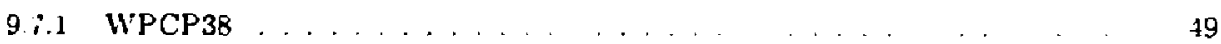




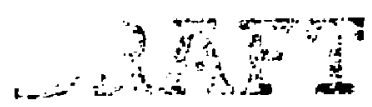

10 Fokker-Planck Codes $\quad 50$

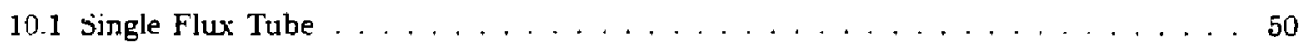

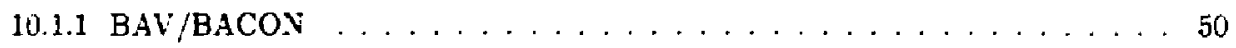

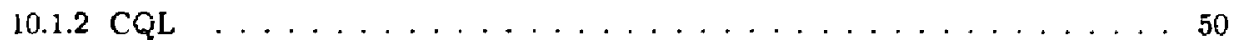

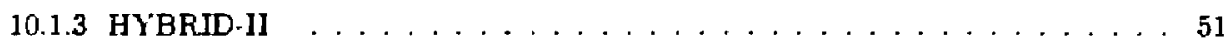

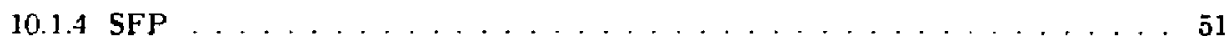

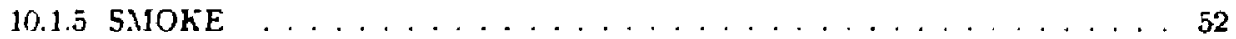

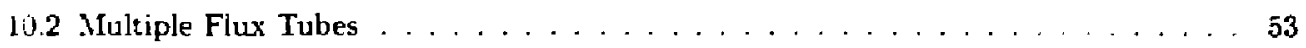

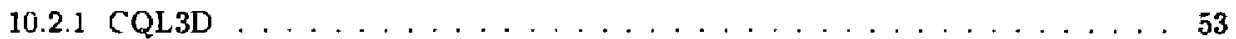

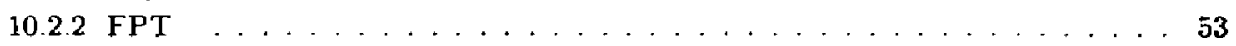

11 Stability Codes $\quad 54$

11.1 Magnetrhydrodynamic Stability . . . . . . . . . . . . . . 54

11.1 .1 FLORA . . . . . . . . . . . . . . . . . . . 54

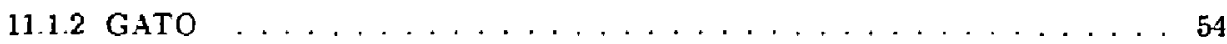

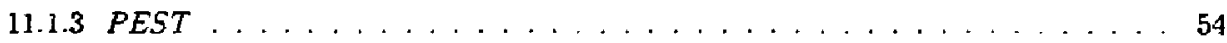

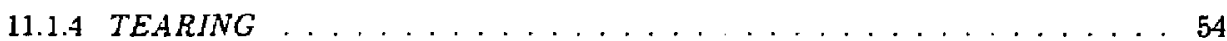

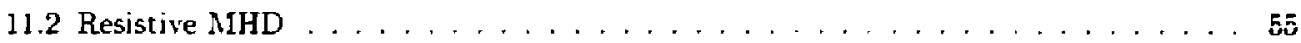

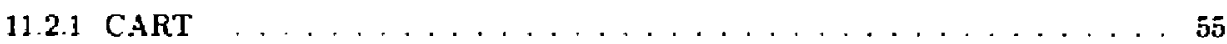

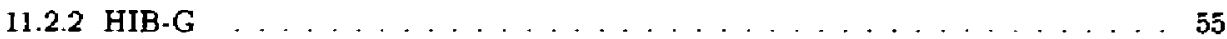

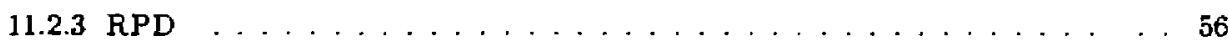

11.3 Mticrostability . . . . . . . . . . . . . . . . . . . . . 56

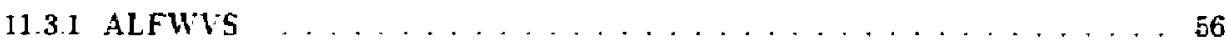

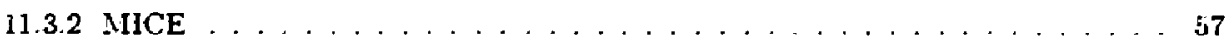

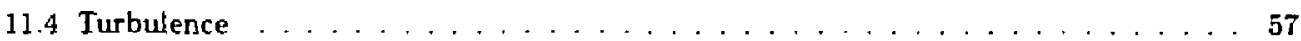

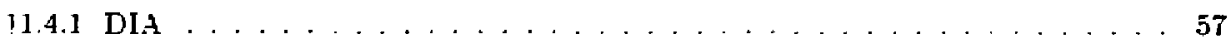

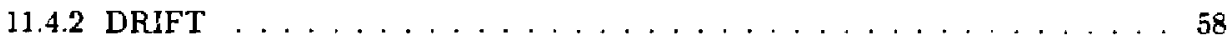

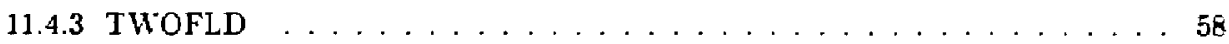

11.5 CROSS-REFEREXCE . . . . . . . . . . . . . . . . . 59 


\section{Introduction}

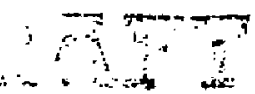

When starting the search for computational tools to address a question about plasma physics, a person may be quickly overwhelmed by the number that are available. This compendium is intended to make it somewhat easier to determine what already exists. I hope that the list of computer codes will enable the experimentalist to find the tools necessary to both thoroughly analyze his data and to determine how those data compare with theoretical models. The National Fusion Program is dorninated by research in toroidal geometry, hence most of the codes listed here are useful for this research. However, some work continues in open geometry, e.g. neutron sources and edge physics st udies, so I have also included those codes that I think will be useful for research in these areas.

Since I am directing this listing toward the needs of the experimentalist, I have tried to provide the information that is likely to be of value to him. Specifically, I expect that he is likely to need several different codes to fully analyze his data. Hence, it becomes valuable to be able to transfer data from one code to another. One powerful tool for this job is described in Sec. 2.1.

Mlany codes are available on the NMFECC's Crays. A deseription of these codes can be obtained on-line 123. I have included some of these codes in this compendium, but by no means all. In general, 1 have tried to provide a summary of the information axailable in Fef. 123. Other code descriptions that are included in this document have been copied verbatim from references describing the codes.

This compendium is intended to be a live document. Computer codes change frequently, and new ones are added regularly. I have written this document using $\operatorname{LAT}_{\mathrm{E}} \mathrm{X}$ to facilitate updates and additions. I anticipate updating the document every few months. Please send information regarding new codes, or suggested changes to G. D. Porter, Lawrence Livermore National Laboratory, P.O. Box 5511. L-637, Livermore: CA, 94550 or via electronic mail to PORTERGLLL.MFENET.

\section{General Utility Codes}

\subsection{B.1SIS}

Description B.ASIS is the name of a system for developing computer programs and running them. It consists of two parts: the development system, which is used to write the user's part of the program: and the runtime system, which combines with the user-written modules to form the complete program. BASIS is designed to assist in the production of programs with sophisticated user interfaces and to solve many of the problems connected with program development, especially for multiple-author programs.

BASIS is the name of the program that results from loading the BASIS runtime ststem by itself. with no user-uritten modules. It is an interpreter for the B.ASIS Language. Like any interpreter. it has enormous advantages over FORTRAX for doing interactive calculations and piots, and for developing and debugging small programs or modules. It is an excellent environment for algorithm development 25 .

A vailability BASIS is a public file and can be accessed by typing basis / $t$ t on the MFECC.

Users A. Friedman, L. LoDestro. G. Smith. M. Rensint, P.F. Dubois. M. Fenstermacher, S.K. Ho. P. Willmann, J. Perkins (LLNL); R.B. Campbell, D.T. Blackfield (Tfll $@$ LL.NL): J. Galambos (FEDC) 


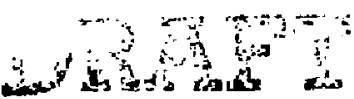

Documentation See Refs. 25,24. To obtain a copy of the most recent BASIS manual type:

cfs filem

read 374 .basisdoc alwith. j2

end

allout nip alwith, $j 2 \mathrm{gr}$, ind. box ann id

while on the MIFECC's Cray

\section{2. $M P P L$}

Description MPPL, (a More Productive Programming Language), is a source code preprocessor. On the Cray's, it is an alternative to precomp. This language allows programmers to write in a c-like language that is more convenient and powerful than Fortran 77. MPPL then transforms statements written in the MPPL language into standard Fortran 77. This language is essentially an extension to Fortran 77 that provides free-form input and many structured const:ucts such as "while" and "for" loops. MPPL's macro preprocessor file-inclusion facility encourages the creation of structured, easy-to-read programs that contain fewer iabels. MPPL provides a more productive programming environment for Fortran 77 users on Unix, VAX and Cray machines.

Special Features MPPL can be used independently as well as with Basis.

Availability MPPL is a public file and can be accessed by typing mppl $/ t \mathrm{v}$ on the MFECC.

Users P. Willman (L.L.NL)

Documentation The MPPL documentation is included in the BASIS manual, which can be obtained as described in Sec. 2.1.

\subsection{GR.AFL-III}

Description GRAFL-III consists of a small set of routines that can produce the vast majority of the figures and plots in the scientific literature. The 38 routines and their arguments have been designed to give the user extensive control over scientific graphs with minimum coding. Versions of GRAFL-III have been implemented on the NMFECC and Octopus computer systems based on the GRAFLIB and TYBOLIB graphics ljbraries developed at $\bar{L}$ LNL. A GRAFLIB version is also resident on the LLL Vax. Although some of the coding uses low-level GRAFCORE routines. GRAFL-111 contains enough comments to permit easy madification or rewriting to drive other graphies libraries. Variations of GRAFL have been written to drive DIGLIB on a Vax, the CERN graphics system and DISSPLA. A version for the Sun is being developed and a GKS version is planned. The NMFECC implementation gives good quality output on every medium - slides, videos, and graphics printers and the 'hardcopy' option provides publication-quality output. The main plotting routines insist on labels to make the output intelligible. These labels can be decoded by GRAFL-1II to produce Greek and math $5 \mathrm{rmbols}$ and to make professional locking scjence graphics. A.l cont rol flags have English names to give clarity to the coding. Alternative numeric values for the flags have been eliminated for simplicity and clarity: Graphs can be produced in many colors simply by sending the out put to the color camera. This process may be expensjue but is useful for presentations. We eliminated interactivity in GRAFL-I11 because the trend is to use test-bed systems such as Basis for developing 


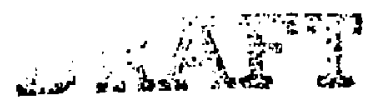

physics codes. These systems already contain sophisticated interactive capabilities. The objective of a good graphics library like GRAFLIB is to provide total control over every pixel of a drawing, whereas GRAFL-III lets physicists and engineers create complete graphs with minimum coding. However, GRAFL-III contains many comments, and most of the interna! parameter choices are in common blocks. These two features permit easy revision for those useis who need more detailed plotting capabilities.

Users Many programs use this graphics package, including BASIS (Sect. 2.1).

Documentation See Ref. 137 .

\subsection{XPOSTPLT}

Description XPOSTPLT is an interactive plotting program for use in conjunction with DDT and its PRINT command. XPOSTPLT can produce plots of either curves or contours from a data file produced by DDT. How to use XPOSTPLT is described in file POSTRPT. The controllee for the CRAY" 2 is called XPOSTB.

Availability The files are in filem 374 .plo

Users P. Willmann (LLNL)

Documentation See Ref. 123

\section{General Physics Codes}

\subsection{Cross-Sections}

\subsubsection{AL.ADDIN}

Description The ALADDIN atomic physics datakase system has been developed in order to provide a standard tool for the exchange of atomic data for fusion applications. ALADDIX is designed to support a wide range of users in the international atomic poysics and fusion modeling communities, and includes a data format definition together with supporting soft ware for both interactive searches as well as access to the dat abase by fusion modeling and other codes. ALADDIN has been accepted as the standard atomic physics data exchange format for fusion applications by the IAEA, which will coordinate entry of critically evaluated atomic data into ALADDIN form.

Special Feat ures ALADDIX is designed to offer maximum flexibility in the choice of data representations and labeling schemes, so as to support a wide range of atomic physucs data types and allow natural evolution and modification of the database as needs change. Support for the widest possible user community was also a prime ALADDIN objective. leading to the use of straightforward ASCII text files with concatenated data entries as the standard data file format, and the adoption of strict FORTRA. 77 code for the supporting softvare. This will allow ready access to the ALADDIX system on the wides range of scientific computers. and easy interfacing with FORTRAX fusion modeling codes, user developed a omic physics codes, databases, etc. The supporting software consists of the ALADDIN interactive searching and data retrival capabilitjes to subroutine 


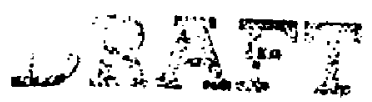

package, which provides ALADDIN datafile searching and data retrival capabilities to user's codes. Associated dictionary files are included in the ALADDIN system for data documentation.

Availability ALADDIN is in its final development _tages, with "beta test" versions of the software and assocjated documentation available from Russell Hulse (PPPL, (609) 243-2621, email HLLSE@PPC. 1 ENET), as well as from the IAEA atomic and molecular data unit (R. Janev, J. Smith). the ORXL atomic data center (R. Phaneuf, et al.), and various international atomic data centers. Final database format and softvare enhancements are presently anticipated for mid-1989 publication and general release. Entry of critically evaluated atomic data sets into ALADDIY format is to be coordinated by IAEA atomic and molecular data unit, which will also coordinate long-term development and distribution of updated software and documentation.

Users Present ALADDIN beta test sjtes include the IAEA atomic and molecular data unit ( $R$. Janev and J. Smith), the ORNL atomic data center (R. Phanuef, et al ), and various international atomic data centers.

Documentation Reference 48 contains the present lAEA atomic data labeling scheme in addition to the ALADDLN presentation. The commented source code and sample data files are also useful for reference. A paper containing a full description of the ALADDIN database format and supporting software is in preparation by R. Hulse for jounal publication.

\subsubsection{SIGI'}

Description SIGV is a modular code on the VAX that is used to compute $(\sigma v)$ for a variet: of interartion pairs involving $\mathrm{e}^{-}, \mathrm{H}, \mathrm{H}_{z}$, He for numerical input with several types of distribution functions.

\section{Special Features}

- Allowance for anisotropic plasma distribution funciion with non-normal bearn injection.

- Estimation of the accuracy of the integration, with the choice of the number of quadrature points then governed by the desired accuracy.

- Provision for use of a 32-point Gauss-Laguerse formula for Maxwellian distribution functions.

- A structure to facilitate modification or incorporation into a larger code.

- A "driver" program that enables the code to be easily run from the terminal of the VAX.

Users R. S. Dewoto (LLNL)

Documentation See Ref. 21. 


\subsection{Veutron and Photon Transport}

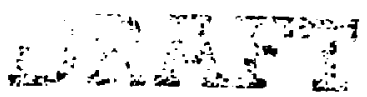

\subsubsection{MCNP}

Description MCNP is a general-purpose Monte Carlo code that can be used for neutron, photon, or coupled neutron-photon transport, including the capability to calculate eigenvalues for critical systems. The code treats an arbitsary three-dimensional configuration of materials in geometric cells bounded by first- and second degree surfaces and some special fourth-degree surfaces (elliptical tori).

Pointwise cross-section data are used. For neutrons, all reactions given in a particular crosssection evaluation (such as ENDF.B- $V$ ) are accounted for. Thermal neutrons are described by both the free gas and $\mathbf{S}(\mathrm{a}, \mathrm{b})$ models. For photons, the code takes account of incoherent and coherent scattering, the possibility of fluorescent emission following photoelectric absorption, and absorption in pair production with local emission of annihilation radiation [123].

Special Features Important standard features that make MCNP very versatile and easy to use include a variety of standard sources, a geometry plot ter, a very rich collection of variance reduction techniques, and etaborate tally structure and user interface that allow a user to easily calculate almost anything conceivable, and an extensive collection of cross-section data. A post processing code, MCPLOT, is arailable to graphically display the MCNP tally output $[123$.

A vailability MCNP version $\mathrm{J}$ is available in FILEM under user 314 in the directories as follows:

\begin{tabular}{|c|c|c|}
\hline directory & name & description \\
\hline \multirow[t]{4}{*}{.crayrun } & manp & executable binary for version 3 \\
\hline & wind & required for plotuing \\
\hline & meplot & executable binary for post prozessor \\
\hline & fr 80 con & converter for FILMIF file \\
\hline \multirow[t]{4}{*}{ crayupd } & $\begin{array}{l}\text { cft } \\
\text { cftlit }\end{array}$ & LANL CFT 110 compiler \\
\hline & $\begin{array}{l}\text { craymath } \\
\text { cgseft }\end{array}$ & LANL libraries needed for MCNP and MCPLOT \\
\hline & $\begin{array}{l}\text { secft } \\
\text { disclib }\end{array}$ & libraries needed for MCPLOT loading \\
\hline & menppl & old program library \\
\hline \multirow[t]{5}{*}{ cravinfo } & $\operatorname{mcnp} 3$ & orjginel source code for MCNP \\
\hline & menplt & source code for mcplot \\
\hline & mcspid & source listing with identifiers \\
\hline & menpix & master index of variables \\
\hline & monpex & LIB file of sample inpur files \\
\hline ver $3 \times s$ & & cross section libraries \\
\hline
\end{tabular}

Documentation See Ref. 123 


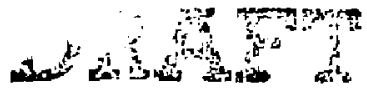

\subsection{Vacuurn Field Calculation}

\subsubsection{EFFI}

Description EFFI calculates magnetic flux lines, fields, forces, and inductance for an arbitrary system of coils made from circular are and/or straight segments of rectangular cross section conductor. This is a packaged code that includes a users manual and sample test cases online i123.

\section{Special Features}

- Auxiliary codes are EJG for generating EFFI input, and EFIMAF for translating EFFI input to MAFCO citenumr:49 input.

- EFFI produces both printed and graphical output. Each page of output is labeled with the problem title, the time, machine, and date of the run, and the version number and compile date for EFFI. In addition, each column of numbers on each page is appropriately labeled Plots of field contours can all be obtained at the option of the user. On field contour and flux line plots, lines of intersection between the coils and the plotting plane are also shown $123^{\circ}$.

Availability The EFFI files are located in FILEW in user number 3011 as follows: filer rds 3011 .effic effi effirpt where EFFI and EFFIRPT are the Cray controllee and users manual. respectively.

Users R. Bulmer (LL.NL)

Documentation See Refs. $123,109,110$.

\subsubsection{GFLY3D}

Description The GFUX3D computer program is primarily a design tool for calculating the magnetic fields for a system of conductors and non-linear (variable permeability) magnetic materials in three divensions. The technique used considers the magnet as a system of known current and unkriown induced magnetization sources. This leads to an integral equation in terms of the unknown magnetization (dipole strength and direction per unit volume). In urder to solve this equation ony the iron needs to be discretised and the need for false boundaries is avoided ' 123 .

Special Features John Kerns has written a program. XFRRI, which converts EFFI coil input into GFL' coil input.

Availability GFLX3D can be found in FILEM as follous:

filem read 3011 .gfunc $x g$ in gfunins.

Users J. Foote (LL.NL)

Documentation See Refs. 123,4. 


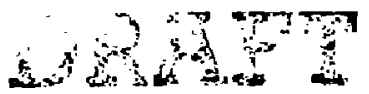

\subsubsection{M.AFCO}

Description MAFCO calculates magnetic field $(\vec{B})$ with filinentary approximation for current elements.

\section{Special Feat ures}

- Input is derived from EFFI input using EFIMAF.

- Fast code for calculating fields away from conductor bundles.

\section{A vailability Contact BulmerOLLL. MFENET}

\section{Users R. Bulmer (LLNL)}

Documentation See Ref. 100.

\section{Data Analysis Codes}

\subsection{General Data Analysis}

\section{i.11. CHIREG}

Description CHIREG is a general purpose regression code for tokamak analysis. To redu e the effects of colinearity, principle components, ridge regression and latent roots analysis are used. Influence diagnostics automatically determine statistical outliers and discard them.

Documentation Results from CHIREG are described in P. McCarthỵ, K.S. Riedel. and O. Kardaun, et al., in progress.

Availability The code is available on request: contact K.S. Riedel; Courant Institute of Mathematical Sciences: New York Lnversity; New York, NY 10012.

\subsection{DATAPLOT}

Description The DATAPLOT language was designed and developed in 19?6 in response to data analvsis protelems encountered in the scjentific/research nvironment at the liational Bureau of Standards. At such time it became increasingly evident that the 3 highest priority computing activities of the scientist/engineer were

1. graphics (especially continuous)

2. fitting (especially non-linear)

3. operations with functions.

DATAPLOT was developed to provide these capabilities 28 
Special Features The language has extensive capabilities in

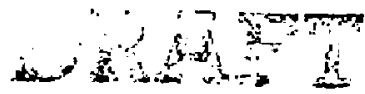

1. graphics (continuous or discrete)

2. fitting (non-linear or linear)

3. general data analysis

4. mathematics

DATAPLOT commands are high-Jevel, English-syntax, and self-descriptive.

A vailability A license for the use of this software can be obtained for a small charge from:

L'S. Department of Commerce

Attn: Waller Finch

Tational Technical Information Service

Office of Data Base Services

5285 Port Royal Road

Springfield, IA 22151

Users G. D. Porter, J. Barter, G. Dimonte (LLYL)

Documentation See Ref. 28.

\subsubsection{IDL}

Description IDL is a software system for the interactive analysis, reduction and display of scientific data. It is designed to help scientists to quickly and accurately analyze their data $56^{\circ}$.

\section{Special Features $[\mathbf{5 6}]$}

- Operatcrs and functions work on entire arrays simplifying interactive analyses and reducing programming time.

- Immediate mode statements provide instant feedback and "hands-an" interaction eliminating tedious edit. compile and link steps.

- The integrated disflay of graphs. curves and images for most popular graphic and image display devices speeds analysis and aid in the understanding of complex data.

- IDL is a complete structured language.

- Programs may be written in any language supported by the VAX/WMS operating system and cabled interartively from IDL.

- Buili-in image: spectral and signal processing capabilities aid the speedy development of applications programs

Availability ILL is available commercially 
Documentation See Ref. $\mathbf{5} 6$.

\subsubsection{LOCtS}

Descriptiun LOCCS is a database system for creating, manipulating. and interactively invest jgating databases. LOCLS was uritten in response to the need to study the data from the experjmental devices at PPPL and is now also used extensively at other fusion laboratories An example of its use is the determination of scaling laws. To do this, LOCLS allous the user to enter and modify data, define subsidiary variables as arbitrary functions of original data, plot and fit such variables, and perform multiple linear regression analysis. Through a flexible jnteractive interface the program is easy to learn and largely self-documenting.

There are two implementations of LOCLS: one that manipulates data in VAX VMS ISAM files and another that manipulates data in the commercial relational database managemras system; INGRES.

Special Features LOCLS allows plotting profiles and time evolving data by specifying the appropriate constraints. It also allows storing frequently used expressions.

\section{Availability Contact MLRPHY@PPC.MFEXET or WIELAND@PPC.MFENET}

Users P.C. Efthimion, R.J. Goidstorı, Hil, J.D. Strachan. Zarnstorf, and many others (PPPL), G.A. Banes, G.A. Wurde?. P.G. Weber (Los Alamos), H.C. Howe. M. Murakomi (Oak Ridge), Bosch (Garching), W.A. Morris, T.E. Edlington (Cuham).

Documentation in Ref. 88, there is an overview of the programs and a users manual for each of the LOCLS versions (INGRES and ISAMI).

\subsubsection{PROFREC;}

Description PROFREG parametrized temperature and density profile shapes in terms of plasma parameters such as line averaged density and edge $q_{\mathrm{a}}$. The normalized profiles re represented by splines or polynomial, with the coefficients being functions of the discharge parameters $a_{t}\left(\tilde{n}_{q_{a}}, l_{p} \ldots\right)$.

The parameteric dependencies are determined by generalized least squares. Spatial correlaijons in the error structure are calculated iteratively. Related SAS routines performed stejowise regressions and casculate influence diagnostics.

Documentaition The method 1 s described in O. Kardaun. K. Riedel. P. MrCarthr, and K. Lack. ner (In preparation). Applications of the code to ASDEX Ohruc profiles are described in $P$ McCarthy; K. Riedel, O. Ka aun. et al. (in preparation).

Availability PFOFREG is avalable on request; contact Kurt S. Riedel: Courant Institute if

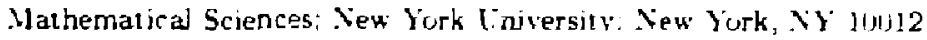




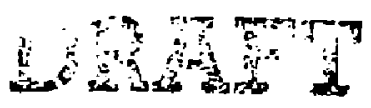

\subsubsection{SIG}

Description SIG is a general-purpose signel processing, analysis, and display program. Its main purpose is to perform manipulations on time- and frequency-domain signals. However, it has been designed to ultimately accommodate other represontations for data such as multiplexed signals and complex matrices. Many of the basic operations one would perform on digitized data are contained in the core SIG package. Out of these core commands, more powerful signal processing algorithrns may be built $69^{\circ}$.

\section{Special Features Two user interfaces:}

- menu made

- command mode

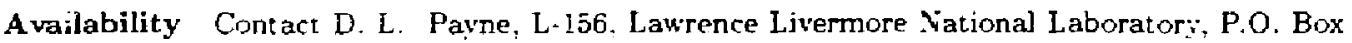
5504. Livermore; CA 94550 .

Users T. A. Casper, G. D. Porter, A. W. Molvik (LI.ML)

Docunentation See Ref. 69

\subsection{Machine Specific Data Analysis}

\subsection{ENERGY'}

Description The ENERGY' code is a diagnostics analysis program used on DIII-D at GA. Given appropriate experimental data the code generates least squares best fit profiles for electron and Ion temperatures. electron densities, radiated power , soft $x$-ray intensity, $Z_{\text {eff }}$, and plasma rotation profiles

Special Features Linear. nonbnear. constrained and non-negative fitting methods aze used to generate the profiles. The profies are assumed to be flux surface functions and are modeled using variable knot cubic spline techniques. Diagnostic dat a can be combined in various wavs to generate new or non-measured data. The code is intended primarjly for interactive use, output is in graphic form.

Availability Information on the techniques used in this code can be oblained from H. St. John at General Atumics.

Users H. St.John (G.1). G. Porter (LL.NL)

Documentation A users manual can be obtained on the General Atrmics Ler Srrtice Center VAX. The numerical techniques used are discussed in Kef. 111. 


\subsubsection{REWEW}

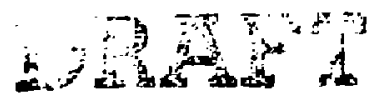

Description REVIEW is a progran io display time traces of data taken on the GA VAX cluster. It can display any pointname versus time in addition to a variety of pseudo-pointnames. These include diamagnetic beta and related quantities as calculated in a simple way from diamagnetic loop data, $\mathrm{CO}_{2}$ interferometer data, charge exchange data, and discharge shape parameters as calculated by the outer flux surface code MFIT 1 i 8

Special Features This is an interactuve code.

Code Output During the run. much of the code output is to the terminal (must be a graphics termunal to be useful). In addition, versatec and/or QMS files can be written.

A vailability This code is used for data analysis on the DII]-D tokamak. Information on the techniques used can be obtained from T. Strait at General Atomics (GA).

Users T. Strait (GA). S. Allen (LLYL)

Documentation See Ref. 118.

\section{Design Codes}

\subsection{Tokannaks}

\subsection{ILMAK}

Description MLMAK is a zero-dimensional (volume averaged), time-independent. power balance code for modeling plasma particle and energy flow in tokamaks. The equations describing the steady-state power balance include terms for alpha power from D-T burning plasmas. inductive (ohmic) as well as non-inductive current drive power, conduction power losses described by empirical energ: confinement scaling law's, and radiation power losses. Fadial profiles of plasma densitv and temperature are controlled by input parameters and are assumed fixed for the priwer balance calculations. MLMAK also performs calculations of non-inductive current drive efficiencies for eleciron cvelotron harmonic (ECH), neutral beam injection (XBI), lower hybrid (LH) slow wave. and fast wave (FW) current drive. The XBI models also include the effects of electron trapping as well as the beam energy dependence. The LH models take into account both the accessjbilitv limit and the condition for awiding st rong electron landau damping of the wave to determine the radius of maximum penetration for the waves $2 i$.

Special Feat ures MtMIK operates in the BASIS code control system (see Sec 2.1).

A vailability Cont act Fenstermacher. user $=31347$ on the MFECC

Lisers L J Perkins, M. E. Fenstermacher. S. K. Ho (LLNL): R. B. Campbell. D. T Blackfield. (TRIVōLYL)

Docurnentation See Ref. 27 


\title{
5.1.2. POPCOX
}

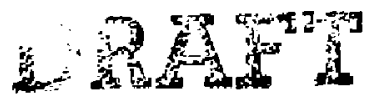

\section{Description}

\section{Special Feat ures}

\section{Code Benchmarking}

\author{
Availability
}

Users

Documentation See Ref. ?

\subsubsection{TADDPACK}

Description The computer code Thermal Analysis Divertor during Disruption PAcKage (TADDPACK) provides three-dimensional graphic presentations of time and position dependent thermal effects on a poloidal cross section of the double-null-divertor configured reactor. These thermal effects include incident heat flux, surface temperature, vaporization rate, total vaporization, and melting depth. 'The dependence of these thermal effects on material choice, pulse shape, and the characieristic thickness of the plasma scrape-off layer is determined ihrough parametric analysis with TADDPACK.

Special Feat ures This computer crode is designed to be a convenient, rapid, and user-friendly modeling tool, which can be easily adajested to most tokamak double-null divertor teactor designs.

\section{Availability Contact L. J. Perkins (LLNI.)}

Users I. J. Perkins(LL.YL)

Documentation See Refs. 13,12.

\section{I.4. TETR.A}

Description The code Tokarnak Engineering Test Reactor Analysis (TH.TRA) consisis of a series of modules. each describing a tokamak system or component, controlled by an optimizer/driver The code has been used to simulate the TIBER II design. A feat ure of this code is the ability to perform optimization st idies through the use of a numerical software package that jterates prescribed variables to satisfy a set of prescribed equations or constrants.

Special Features This rode operates under the BASIS code control sy'stem (see Set 21 ).

Availability To obtain on the MFECC, contact J. D. Galambos (FEDC), or L. J. Perkuns (L. IL) 


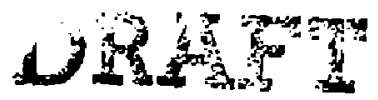

from the numerical computation of the eigenfunctions of the bounce-average Fokker-Planck equation. (J.G. Cordey, 19.). Seoclassical currents come from the expressions in the review paper of Hinton and Hazeltine 41 . The MHD equilibrium is made self consistent with the driven current in an iterative process. It has been used to simulate experiments in DIII-D and to predict performance of ITER when operating in steady state. It is currently being modified to include lower hybrid current drive.

A vailability Permission to obtain copies of this code must be abiained from JAERl.

\author{
Users R. S. Devoto (LLNL)
}

Documentatiors See Refs. 120,22.

\title{
6.2.2. $A D$
}

Description $A D$ is a large class of codes employing Grad's technique of iterative aiternation between solving for the 2-D or 3-D geometry of flux surfaces and for tansport as defined by a set of profiles. The codes are applicable to slow-evolution problems for which the time to equilibrate within flux surfaces is short compared to the time scale of the phenomena studied. They solve the fully ronlinear macroscope MHD equations dropping the momentum term, either with dissipation 32 or adiabatic 33: An important feature of AD codes is their ability to handle complicated topologies and free boundaries with high precision.

The most fully elaborated code of the AD family is the $3 D$ code employing anisotropic Braginskii transport 8. This code (and a non-Braginskii) variation has been applied to study the "anomalous" coupling of plasma diffusion and heat flow $44,45^{\circ}$. Kress '68] has derised and implemented techniques to accelerate convergence in the low resistivity regime. The following $2 \mathrm{D}$ and axisvmmetric implementations of $A D$ are referenced in $34:$

- 2D adiabatic Tokomak. Belt Pinch, and Doublet

- Axisymmetric adiabatic Doublet

- 20 adiabatic tearing (Lens)

- 2D resistive Tokomak and Doublet

- Livertor

- Mirror Machine. Simple and Reversed Field.

A vailability All codes are available on request: contact $\mathrm{M}$. Kress and A. Blank: Magnetofluid Dynasnirs Djuision: Courant Institute of Mathematical Sciences: Yew York Lnviersity, New York. CY' $1(11) 12$.

Documentation See Refs, $32,33,8,44,45,68$, and 34 . 


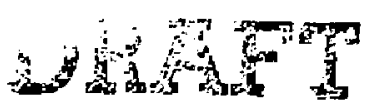

\subsubsection{BETA}

Description The BETA code is a well known research tool for two- and three-dimensional magne:ohydrodynamic equib brium. MHD equilibria are calculated in flux coordinates using an accelerated iteration procedure. The nonlinear stability of this equilibrium is analyzed using a variational procedure.

The BETA code has been extensively used in the design of all major stellarator experiments. Tokamak applications include modeling magnetic field ripple, helical shaping coils. and divertor configurations.

Availability The BETA code is currently listed on the NAIFECC ai Livermore.

Documentation All aspects of the code are described in ' 5 . and i 6 ;.

\subsubsection{EFIT}

Description The equilibrium-fitting code EFIT was developed to perform magnetic analyses for Doublet III. It can be run in either the fitting mode or the equilibriur ! mode. EFIT exists on the GA VAX's and the MFECC. In the equilibrium mode, it acts as an equilibrium solver. It the fitting mode, it performs magnetic fittings. The magnetic data needed for fitting can either be retrieved from the data bank directly or from an input file. There are two versions: EFIT for Doublet III (D-III), and EFITD for Doublet III D(DIII-D). Additionally, EFIT can also perform fixed boundary calculations '72:.

Users L. Lau (GA); G. Porter (LLNL)

Documentation See Ref. 72.

\subsubsection{EPFC}

Description EPFC calculates an approximate fixed boundary toroidal equilibrium using varjational techniques. Sisgle null, double null, and linited tokamak configurations with finjte aspect ratis and arbitrary' $p^{\prime}$ and $F F^{\prime}$ profiles can be modeled. A version of the code capable of handling RFP configurations also exists. Despite the approximate nature of the method. fiux surface topolog: along with local and integrated plasma parameters computed by EPFC have been shoun to agree well uith those computed by exact equilibrium codes (even for strongly shaped plasmas as is found for ITER).

Special Features $T h\lrcorner$ code is quite fast. Execution times on various computers include: Crav2-0.01 - 11.06 sec, Vax 11/780-0.5 - $2.0 \mathrm{sec}$, Vacintosh II-4.0 - $15.0 \mathrm{sec}$.

A vailability EPFC currenty runs on Cray, Vax, and Macintosh computers. Cuntart 5 . 14 Haney (A1FECC user 3U273, (415)422-6715) for copies of the code.

Users S IV Haney (LLNL)

Documentation A manual accompanies the code. 


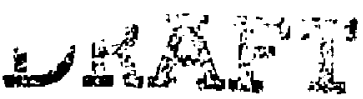

\subsubsection{EQPD}

Description EQPD finds axisymmetric MHD equilibria in non-diverted and four-node poloidal divertor tokamaks with square cross sections. Uses the 2-D Chodura-Schlïter method in $\because$ hich 2-D magneto-hydro-friction equations are advanced numerically with conjugate gr:dient term. $\mathrm{Cp}_{\mathrm{p} \text {-down }}$ symmetry assumed. A small but finite resistivity introduced to obtain smooth plasma profiles near the divertor separatrix and internal divertor rings. Can change the number of divertor rings with moderate amount of code modifications. The code used primarily to iritialize tire 3-D resistive MHD simulation code, RPD, but can be used as the basis of transport calculations in poloidal divertor as well as non-diverted tokamaks.

Code Developers Eijiro Lichimoto (Courant Institute, Jew York University), and James D. Callen (Cniversity of Wisconsin-Madison).

Special Feat ures Language: FORTRAN77. Compiler: CIVIC or CFT77.

Documentation E. Lichimoto, Ph.D. Thesis, Dept of Physics, Unix. of Wisconsin-Madison (1988).

A vailability Contact Ejjiro Lchimoto, Courant Institute, Sew York Lniversity, 251 Mercer St. New York, NYं 10012; Tel. No. (212)-998-3273: MFE user No. 1556.

\subsubsection{YEQ/HEQ/VEQ}

Description These codes solve the equilibrium equations for axisymmetric $(R-Z)$. free boundary problems. The output includes an eqdsk file suitable for input to stability codes. NEQ solves for poloidal field coil currents required to achieve zero pressure at a number of specifiable points with optional coil current minimization. HEQ solves in addition for coil currents and profile parameters required to $\mathrm{fx} X$-point or limiter strike point locations and volt-seconds. $\mathrm{V} E \mathrm{Q}$ can in addition minimize the magnetic energy. TEQ is a BASIS (see Sec. 2.1) version of these codes. It allows a very flexible runtime specification of the equation set. can do multiple runs; and includes all the $\mathrm{N} / \mathrm{H}$ : $\mathrm{EQ}$ features.

Availability On the MMFECC the files needed 10 run $\mathrm{HEQ}$ can be obtained from FILEMI in directory heqpub of user 1054 . Contact Dennis Strickler to obtain XEQ or IEQ. To obtain the LLNL version (TEQ), contact R. Bulmer (bulmer $\bar{g} l l$.mfenet).

Users D. Strickler (ORXL), L. L. LoDestro (LLNL).

Documentation See Ref. 119.

\subsubsection{1:MEC}

Description 'MEC' is a code that solves for .MHD equilibria in toruidal plasmas (t.,kamaks. rippled tokamaks, stellarators, torsatrons. and heliacs) 


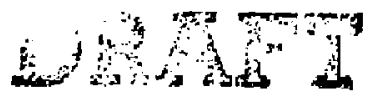

Special Features VMEC uses a steepest descent mornents approach to determine the spectra] representation of $\mathbf{R}, \mathbf{Z}$, and a reicrmalization parameter for the poloidal angle. It can be run in both free-boundary and fixed boundary modes. Options exist to specify either no net current (steady-state stellarator condition, solve for the rotational transform) or the rotational transform profile (solve for the net toroidal current). Options also exist for specification of the adiabatic pressure constraint. At present VMEC cannot handle magnetic islands.

Integration with Othes Codes VMEC has been used in 2-D form as a module in the TRAXSP transport code (Sec. 8.1.14) and is being incorporeted into the WHIST transport code (Sec. 8.1.16). The TRACK code (Sec. 8.1.12) can be used to evaluate geometric information from the spectra] coefficients. The STELOPT code uses VMEC in an optimization loop to obtain configurations with suitable transport and stability properties.

A vailability A stand-alone version of VMEC demonstrates some of its capabilities and is available in either VAX or Cray forms. The stand-alone version uses DISSPLA graphics. Contacts at ORXL (FTS prefix 624): S.P. Hirshman (615)-574-1289, NMFECC user 251; W.I. Van Rij (615)574-0639, NMFECC user 27770; Stan Attenberger (615)-574-0613, NMECC user 21070; Wayne Houlberg (615)-574-1350, N.MFECC user 2357.

Users ORXL: S.E. Attenberger, N. Dominguez, S.P. Hirshman, L.M. Hively, W. A. Houlberg; D.K. Lee, R.X. Morris, W.I. Van Rij. PPPL: D.C. McCune, R. M. Wieland.

Documentation See Ref. 43 .

\subsubsection{IMOMS}

Description THOMS is a code that solves for fixed boundary MHD equilibria in tokamak plasmes.

Special Features MOMS uses a shooting method to determine the spectral representation of $\mathbf{R}$ and $Z$, and assumes up-down symmetry Results are presented in terms of the shift. elongation; and triangularity. The toroidal current profile and plasma presure must be specified. VMOMIS cannot handle magnetic islands.

Integration with Other Codes VMOMS has been used as a module in the WHIST (Sec. 8.1.16) and PROCTR (Ser. ??) transport codes. The TRACK code (Ser. 8.1.12) can be used is evaluate geometric information from the spectral coefficients.

A vailability A stand-alone versicn of VMOMS demonst rates some of its capabilities and is avajlable in either VAX or Cray forms. The stand-alone version uses DISSPl.A graphics. Contacts at OKXL (FTS prefix 624): Stan Attenberger (615)-574-(1613. XMECC user 2lu70: Warne Houberg (615)-57t-135u. XMFECC user 2357.

Lisers ORNL: W.A. Houberg, E.A. Lazarus, W.I. Van Rij.

Documentation See Refs. $73-75,71$. 


\section{Particle Following Codes}

\subsection{Single Particle}

\subsubsection{TIBRO}

Description TIBRO follows single particles in magnetic fields computed with MAFCO-style flamentary approximation.

Special Feat ures Long-used code (mature).

Availability The Cray source, compiled version, data, examples, and description may be obtained from Steve Devoto on the Cray by typing filem rds . 317 , tibroc tibro. The file tibro is a bib file containing all relevant files.

Users R. S. Devoto, J. H. Foote (LL.YL)

\section{Documentation $3 \overline{5}_{j}$}

\subsection{Monte-Carlo}

\subsubsection{MCGO}

Description MCGO follows single charged particles in 3-D magnetic fields either supplied analytically or on a grid. When supplied on a grid; the fields are represented by tri-cubic spline coefficients. Orbit code for studying fast ion behavior in tokamaks. Individual particle orbits may be calculated from appropriate initial conditions. Neutral beam injection may be sirnulated with NFREYA (Sec. 9.3.1) and the resulting steady-state, fast-ion distribution is obtained. Magnetic equijbria are specified with either concentric circular models or from EQDSK-type axisymunetric equilibrium data files. Processes simulated include, finite banana-width, charge exchange (primary: secondary, and resonant), electron and ion drag, energy diffusion, and limiter impact.

Code Output Extensive graphical output is generated. Time-dependent quantities (e.g. B. $v$. Vi) as well as orbit projections are depicted for single particle orbit calculations. Representations of neutral beam deposition and steady state fast jon distribution (e.g. heating, current, pressure) are determined in the neutral beam mode.

Availability Contact Glenn Sager (619) 455-3789 or R. W. Harvey (619) 455-3761 at General Atomies (GA).

Lisers G. Sager (GA), A. Molvik (LL.iL)

Documentation Some documentation is self-contained in the source. 


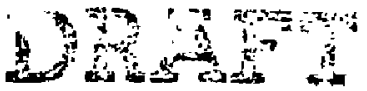

\subsubsection{MCPAT}

Description MCPAT, a Monte Carlo code for Particle Axjal Transport, evolves an ensemble of test electrons or ions in prescribed axial electric and magnetic fields using relativistic guiding-center equations of motion [106]. The particle gyrophase is calculated, and if heating can be modeled directly by equaticis of motion 106? or by using a quasilinear model. Collisions with electrons and ions are modeled by a Monte Carlo technique where Maxwellian background distribution functions with varying densities and temperatures are specified, and Spitzer scattering coefficients are used '108. The code usually solves steady-state problems where the profiles of the background distributions may be updated from the test-particle information by a iteration procedure. The code can also be run in a quasi-time-dependent mode where an ensemble of particles are followed for a moderace number ( 10 to 50 ) of macro time-steps. The code's primary use is in calculating density and energy profiles along a magnetjc field line either in open-end geometry or in periodic geometry for toroidal devices. The code has been used to study parallel electron heat transport in a tokamak divertor region when the mean free path is comparable to the distance to the divertor plate. Electrostatic potential variations can be determined by as: ining a Boltzmann distrjbution of electrons for test ion problems or using ion fluid equations for test electron problems.

\section{Special Feat נres}

- MCPAT uses magnetic field profles generated by EFFI or from a cubic spline representation.

- The code can be restarted so an iteration series can be continued.

- Improved efficiency is provided by particle splitring techniques and by accelerated collisional and if diffusion processes.

- Output is available on an FR80 plot file, which gives test-particle profile data and momentum space distribution functions. The confinement time and energy flow are also given. A printed output file has more detailed diagnostic information.

\section{A vailability Contact RognlieneLLL. MFENET}

\section{Users I. D. Rognlien (LLNL)}

Code Beuchmarking The code has been benchmarked on a number of problems. The collisjonal relaxation of test particles to a background Marwellian has been verified. It yields the Pastukov rate for long mear1-free-path confinement in a magnetic mirror and flow confinement for a short mean-free-path. Spitzer thermal conduction has been reproduced for a highly collisional plasma. The calculation of $\mathrm{r} f$ heating using the equations of motion gives the same results as the quasilinear model in the appropriate parameter regime, and super adjabatic reduction of $r$ heating agrees with theory for a simple quadratic magnetic well. With the quasilinear model of $r f$ heating the code compares well with Fokker-Planck codes

Documentation See Refs. 106,108. 
7.3. Particle-in-cell Simulation codes with self-consistent fields

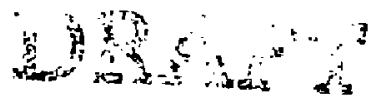

\subsubsection{D.ART}

Description This is a recently modified version of the DART cade designed to simulate the behavior of a beam of charged particles whose paths are affected by electric and magnetic fields. This code is used to design laboratory scale and full-scale beam direct converters. The code in its modified version has been generalized to treat a wide variety of problems in addition to direct converters. The simulation technique includes space charge. secondary electron effects. and neutral gas ionization, and charge exchange. Calculations of electrode placement and energy conversion efficiency are described 136 .

Availability Contact Moirelil. MFENET

Users R. IH. Moir (LLKL)

Documentation See Ref. 136.

\subsubsection{DIPSI}

Description DIPSI is a $1 \frac{1}{2}-\mathrm{D}(1 \mathrm{D}, 2 \mathrm{~V})$ electrostatic PlC code that is applied to the study of plasma-surface interactions in bounded plasma systems, such as divertors or plasma et ching sy'stems. DIPSI was derived from the TESS code (Sec, 7.3.8).

\section{Special Feat ures}

- Planar or Volume Suwce Partirle Injection

- Particle abstorption or reflection at the collectur plate

- Coulomb Collisirin Misdel

\section{A vallability Contact Prucassini @LLL.MFENET}

\section{Users R.J. Procassind (CCB)}

Documentation See Refs. 126.18.

\section{-3.3 E.MOYE}

Description ENOXE is a $1 \frac{1}{2}-D$ relativistic, electromagnetic PIC code

\section{Special Features}

- parame1 ric instabilities

- KF heating

- current drive 
Users B. I. Cohen (LLNL)

Dacumentation See Refs. 1T,10.

\subsubsection{ESI}

Description ES1 is a basic 1-1/2 D PIC many-particle code, periodic, can be magnetized or not, with a great variety of initial conditions for $f\left(x, v_{x}\right)$.

Special Features A PC version of this codes is also available.

Availability Contact C.K. Birdsal at L'C, Berkeley: MFE user number 1200.

Users C.K. Birdsal (LC Berkeley)

Documentation See Ref. 10, Chapters 1-5.

\subsubsection{PDI1}

Description PDWl is a 1-1/2 D PIC many-particle bounded code, electrodes at either end, witt: external circuit.

PDW1 operates on the principles espoused in Ref. 10 with sorre additional features described in Fef. $i \bar{i}$. Ljection of particles from the boundaries is supported, as is initial loading, for a drifting Maxuellian distribution. (The injection parameters for PDW 1 are actually painfully tomplete, so a modification called FDWMAK is also available for those who don't need the full generality) Other distributions and particle sources are minor modifications. The extemal circuit is a serjes RLC circuit with both AC and DC voltage sources. A constant magnetir field in any direction is allowed.

Transportability has been made as easy as possible by limiting system-dependent subroutine calls (such as graphics and other 1/0) to a very few of the main routines in the code. The only compiler-dependent features are the use of a precompiler and one use of a compiler directive. These should be east to translate to another system.

This cide was intended for use both as a research and teaching tool. Because of this. it was written with srme care toward clarjy and, therefore, should be relatively easv to learn and use.

\section{Special Feat ures}

- elect rodes emit drifting cut-off Maxwellian velocities

- electrodes may be absorbing, reflecting, secondary emitting, etc.

- useful for l-D sheath particle and energy transport

- PC versiur also available 


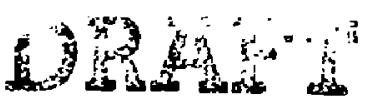

Availability Contact Willian Lawson, Courant Inst.. 251 Mercer St., Yew York, Ny 10012 , (212)-998-3260: Prof. C.K. (Ned) Birdsall, EECS Dept., U. of Calif., Berkeley, CA 94720, (415)643-6631; Perry Gray, Dept. of Physics, Dar:mouth College, Hanover, NH 03755, (603)-646-3969

Users C.K. Birdsall (UC, Berkeley)

Documentation See Refs. 10,76, and 77 .

\subsubsection{PDII2}

Description PDW2 is a particle, electrostatic, 2-1/2 D, bounded in $\mathrm{X}$ with external circuit, periodic in $Y$ code.

PDW2 is intended as a directly upward compatible two dimensional extension of PWD1. PDW2 operates on the principles espoused in R.ef. 10 with some additional features described in Ref. 77. Fields are solved with a combined finite difference Fourier technique with rectangular grids [10'. Injection of particles from the boundaries is supported, as is initial loading, for a Maxwellian distribution. Other distributions and particle sources are minor modifications. The external circuit, in the bounded dimension, is a series $\mathrm{ALC}$ circuit with both $\mathrm{AC}$ and $\mathrm{DC}$ voltage sources. A constant magnetic field in any direction is allowed.

Transportability has been made as easy as possible by limiting system-dependent subroutine calls (such as graphics and other $I / O$ ) to a very few of the main routines in the code. The only compiler-dependent features are the use of a precompiler and one use of a compiler directive. These should be easy to translate to another system.

This code was intended for use both as a research and teaching tool. Because of this, it was written with some care toward clarity and, therefore, should be relatively easy to learn and use.

Availability Contact Perry Gray, Dept. of Physics, Dartmouth College, Hanover, NH 0375̃̄, (6u3)-646-3965: Prof. M.K. Hudson, Dept. of Physics, Dartmouth College, Hanover, МH 0375ā, (6r3)-646-2976; Prof. C.K. (Yed) Birdsall, EECS Depc., L. of Calif. Berkeley: CA 94720, (415)643-6631: William Lawson, Courant Lnst., 251 Mercer St., New York, NY 10012. (212)-998-3260.

Users C.K. Birdsall (LC, Berkeley)

Documentation See Ref. 76.10 .57 .

\subsubsection{PDIIZ}

Description PDWZ is the same as PDWl (Sec. 7.3.5) but in $2-1 / 2 \mathrm{D}$, where $x$ is the bounded direction and $y$ is the periodic direction.

\section{Special Features}

- allows wave along bounding walls, like Kelvin Helmholtz instability for magnetuzed plasma (see PKL 13. Feb, 1989). 


\subsubsection{TESS}

Description TESS is a $1 \frac{1}{2}-D$ guiding center, open-field line electrostatic PIC code.

\section{Special Features}

- ECRH

- ICRH

- Seutral beam injection

- Ambipolar potential

- Coubomb Collision Model

A vailability Contact BCOHENeLLL. MFENET

Users B. I. Cohen (LLNL), R.J. Procassini (LCB)

Documentation See Refs. 126,18.

\subsection{ISI}

Description VSl is a basic 1-D electsostatic code in which the perturbed part of the Vlasov equation is solved along the particle trajectories. The Vlasov equation may or may not be linearized. The code has reduced particle noise, especially in the linearized case.

Availability Contact E. Morse; MFE user number 3015,5

Users G. DiYen. L.C. Berkeley

Documentation Forthcoming.

\subsubsection{ZOHAR}

Description $2 O H A R$ is a $2 \frac{1}{2}-D$ relativistic, electromagnetic PIC code.

\section{Sperial Features}

- Parametric instabilities

- HF heàung

Availability Contact BCOHEHOLLL. MFENET

Users A. B. Langdon, B. I. Cohen (LJ..IL) 


\section{Documentation See Ref. 70 .}

\section{Plasma Transport Codes}

\subsection{Cross-Field Transport}

\subsubsection{B.ALDLR}

Description BALDLR is a $1 \frac{1}{2}-\mathrm{D}$ transport code designed to simulate a wide variety of plasma conditions in tokamaks. The code follows the time evolution of particle densities (up to two hydrogenic species and up to four impurity species), electron and ion temperature, and poloidal magnetic flux density as a function of flux surface. The shapes of the flux surfaces are determined by solving axisymmetric equilibrium force balance equations. given conditions that may be changing with time. BALDLR provides a detailed treatment of neutral hydrogen transport. auxiliary heating, mult ispecies effects (including an extensive atomic physsics package); orbits of $\alpha$-particles, plasma compression, ripple transport, and edge processes such as "scrape-off" losses. There are a wide variety of transport models available as options, and different transport models can be combined additively. In addition, there are various options to treat the axisymmetric effects resulting from sawtooth oscillations, saturated tearing modes, and high-n ballooring modes. The code has been used to aid interpretation of data from tokamak experiments and for evaluating designs of future tokamak devices.

Special Features Most of the BALDLR code follows OLYMPLS conventions. The 1-D version of the code used the OIYMPUS preprocessor. XAMELIST is used for specifying the input parameters, with a provision for inline comments in the input file. Choices among a variety of graphical and printed output options are provided.

Availability Contact Redi@PPC.MFENET or Bateman@PPC.MFENET. Recent versions of the code are available on the LS Magnetic Fusion Energ. Computer Center. The 1-D version of the code described in Ref. 114 is in the CPC Program Library: Queen's Lniversity of Belfast, N. Ireland.

Users 11 Redi, Glenn Bateman, D. P. Stotler (PPPI), C. E. Singer (L: Dinois). R. Englade (.МT), M. Hughes (Grumman), S. Sabbagh (Columbia)

Documentation $A$ 1-D version of the code is documented in Ref. 114. Additional di.cumentation Fior the $i \frac{1}{2}-D$ version of BALDLR is available online at the LS Nagnetic Fusiun Energy Computer Center.

\subsubsection{BETA MC (Monte Carlo)}

Description The BETA MC code calculates particle and heat losses through Monte Carlo simulations of particle orbits. The guiding centers of particle trajectories are calculated in Bouzer conrdinates and discrete particle effects are simulated using a model collision operator. The elecisic-field potential is calculated self-consistenty from quasineutrality instead of Ohrn 's law.

The BETA MC code uses a number of advanced numerical techniques that significardy enhance its performance abrse the standard scheme. The input data is automatically produced in the BETA equilibrium ecode 


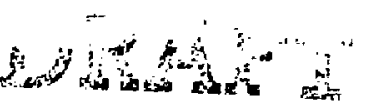

The major physical results is that the electron-loss rate becomes comparable to the ion-loss rate. Magnetic field ripple and electromagnetic perturbation produce this effect in tokamaks. To model three dimensions and perturbations in tokamaks, small helical perturbations have been added to the plasma.

A vailability The BETA MC code is avajable on request: contact Frances Bauer, Octavio Betancourt and Paul Garabedian: Courant Institute of Mathematical Sciences: Yew York Universicy: Sew York, XY 10012.

Documentation lt is documented in $77^{*}$.

\subsubsection{CFRX}

Description CFRX is a 1-1/4-D fluid transport code designed to simulate a wide sariety of plasma conditions in the Field-Reversed Configuration (FRC). The code follows the temporal evolution of ion density, ion and electron temperature, and axial magnetic field as a function of flux surface in both the closed- and open-field-line regions. The FRC equilibrium is assumed to be highly elongated: and equilibrium is enforced through a radial pressure balance equation and a radialiy-averaged axial tension balance constraint. Cross-field transport is followed using classical or Bohm transport scalings. The extra $1 / 4$ dimension arises through the inclusion of three multidimensional effects: (1) flux-surface averaging within the closed-field region: (2) axial contraction of the closed-field region mandated by the equilibrium constraints; and (3) parallel transport loss terms included on open field lines. CFRX has been used to aid interpretation of data from FRC experiments at Los Alamos.

A vailability The code is available on the LS NMFECC letwork, contact $k-M$. Ling, $k$.A. Werlev (LANL) or M.-Y. Hsiao (Penn State). The code will also soon be atailable in the CPC Program Library, Queen's Lniversity of Belfast, $\mathrm{X}$. Ireland (Ref. 47).

Users K.-1. Ling, D. Rej, K.A. Werley (LANL). M.-Y. Hsiao (Penn State)

Documentation The formulation of the model is described in Ref. 15. The numerical embodiment and application of the model are described in Ref. 135. A user's guide is provided by Ref. $t i$.

\section{L.A CHAPO}

Description (HAPO is a predictive one-dimensional transport code. It has several modules that can be activated or deactivated individually. The primary module integrates the radial diffision equations for the parallel current. the electron and ion energies, and the density of the working gas. These is a neutral particle module and two impurity modules, each of which allows for al the charge states of an impirity element to be evolved. The code is designed for the comparison of theoretically based transport mudels with experimental data in a context that is geometricallv sumple, but includes a description of all relevant subsidiary physics (sources and sintis).

Special Features Implementation of a large number of theoretical models. including ff-diag nal element s: links to the TEXT and MFE experimental databases; flexibility and ease of use. 
Availablity Contace James (". Wilev. Fusion Research Center. The Lniversity of Texas at Austin. NileyatTA

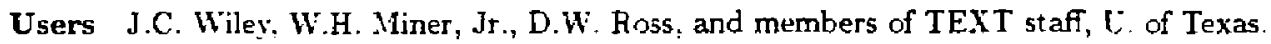

Documentation Adraft report describing the code has been prepared. $I$ is arailable to prospectite users upon request.

\subsubsection{HPFE}

Description The Heat Pulse Forced Boundary (HPFB) is a predictive transport cade used to ralculate the thermal diffusivity from time-dependent perturbations. The advantage of HPFB over traditional predictive codes is that the actual experiment ally measured diagnostic data is input into the computation through time-dependent boundary conditions. No theoretical assumptions about input power or thernal diffusivity in the region outside the heat pulse tegion are necessary; thus. a more accurate determination of $\chi_{e}$ is possible.

Buth convective and diffusive terms are included in the density and electron-and-ion temperature equatirns. Neoclassical resistivity and ion heat conductivity are used. To reduce experimental noise. a sant tooth-averaging model is included.

Documentation The method and results obtained in ASDEX are documented in Ref. 104.

Availability Copies of the code are available on request: contact Kurt S. Riedel, Courant Institute of Mathematical Sciences, New York Lnversity, New York, NY 10012: and L. Gianone. Max-Planck Institute for Plasma Physics, Garching: West Germany.

\section{J.6. JHT.YDC:}

Description IHTIIDC is a 2-dimensional heat transport code for calculating ion heat transport in a model divertor configuration. The geometry is simplified by replacing the true magnetic-divertor geometrv with a circular cross-section model and a toroidaly symmetric limiter. The jon-energy balance equation is solved for the ion temperature in a boundary laver containing the separatrix. defined as the magnetic surface that is tangent to the limiter, neglecting particle transport and energy: exchange with electrons. Both neoclassical and anomalous transport are included. The standard neoclassical transport is generalized near the separatrix to include the significantly larger polojdal iemperature gradient there. The anomalous transport is modeled with a larger poloidal temperature gradient there. The anomalous transport is modeled with a simple term containing an anomalous thermal conductivity whose value is an input parameter. The ion temperat ure and the necoclassical and anomalous heat fluxes, averaged over a magnetic surface are oblained as functions of munor radius. for various values of the input parameters, which include the temperature gradient well inside the separatrix, the magnitude and polojdal dependence of the anomalous thermal rondurturits, the aspect ratio, and the poloidal field of the divertis

A vailability ('ontact F.L. Hintron at General Alomics (Ci).

Users F L. Hinton 


\subsubsection{MINERIA}

Description MINERVA is a 1-1/2 D transport code designed to simulate low beta Spheromak transpcrt. The separatrix is allowed to touch the geometric axis and special attention is given to including the resultant singularities correctly. The code can presently calculate the magnetic field diffusion (which decouples from the equations for the evolution of the density and temperatures). Classical transport is assumed, but not hardwired in. Other transport models may be included as subroutines. Inclusion of the evolution of the density and temperatures is underway.

A vailability The code is presently under developinent. An experimental version (no guarantees) may be had from $517 \mathrm{mfe}$.

Users A.G. Sgro

Documentation See Ref. 112.

\subsubsection{MIRTH}

Description MIRTH, (Multigroup Ion Radial transport and THermalization), is a code that solves an approximation to the Fokker-Panck equation appropriate for thermaljzation of fast jons from neutral beam injection or fusion reactions.

Special Features The MIRTH code uses a simple approximation to the time-dependent FokkerPlanct equation to calculate downscatterirz in energy. The pitch angle dependence is integrated out of the equations. The dounscattering includes that due to classical Coulomb collisions plus an option for a user-supplied energy loss term. Another option exists to jrajude radial diffusion in read space replacing the usual pitch angle terms.

Integration with Other Codes MIRTH is a module of the WHIST (Sec. 8.1.16) transport code. and is used for both fast beam ions and fast alphas.

Availability Contacts at ORNL (FTS prefix 624): Stan Attenberger (615)-5i4-0613. NMFECC user 2107u: Wayne Houlberg (615)-574-1350, NMFECC user 2357.

Users ORNL. S.E. Attenberger, W.A. Howberg.

Documentation See Ref. 1.

\subsubsection{O.ETIIO}

Description ONETMO is the 1-1/2 D time-dependent main transport rode developed and used at General Atomics sine the mid-70's for modeling plasmas in tokamaks. Both circular and noncircular cross section plasma can be modeled with specified currents in field shaping coils. Electrons and several jun species are treated as furds and neutrals are treated kineticalls. Neoclassical and 


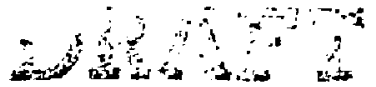

model anomalous transport mechanism are treated. Important physical process that produces sources and sink $2 \Gamma$ particles and energies are treated with various subroutines: NEC CG for wall recycled neutrals. NFREYA (Sec. 9.3.1) for neutral beams, TORAY (Sec. 9.1.2) for electron cyclotron heating and current drive to name a few examples. Models for pellet fueling, fusion. ohmic heat ing fast ion to name a fow examples. Models for pellet fueling, fusion, ohmic heating fast ion storage are also included. Short circuit transport processes for sawteeth and low'm tearing modes (DELSOL) ?? are included.

There are t wo ways of treating MHD equilibria: A ID mode in which simple ellipsoidal flux surfaces or externally generated and arbitrary equilibria are used: and a 1-1/2 D mode in which magr, stic flux surfares are determined and updated with calls to a $2 \mathrm{D}$ MHD equilibrium code.

Special Features There are two modes of operation. In the simulation mode the primary quantitjes such as particle densities, temperatures and current density are transported with prescribed diffusion coefficients using the particle and energy conservation equations and Faraday's law. In the analysis mode experimental density and temperature profiles are inverted from calculated sources to find the diffusion coefficients.

Code Output The code output is both numerical and graphical. Graphical output is created by ruming post-processors on files created during the run. Separate processors are used for equilibrium. transport. and neutral beam plots. Control of the quantities actually plotted is provided by plot flags and scale parameters in the input file.

\section{A vailability Contart H. St. Johs at General Atomics (GA) StJohmeGAV .MFENET}

Users R.E. Maltz. R.R. Dominguez. R. Stockdale, R. W. Harvey; K. Matsuda, K.H. Burrell, D.P. Schissel, R. Groelsner (GA): G.D. Porter (LL.NL): and S. Wolfe (MIT).

Documentation The code is described in Ref. 101. Sample publications of work done with the code include Ref5. 23, 94, and 39.

\subsection{JU. RFPBLRA}

Description RFPBLHX is a 1-D (cylindrical) transport code designed to simulate a wide variesw of plasma conditions in the Reversed Field Pinch (RFP) and in the High Density Z-Pinch (HLZP). The code follows the radial and temporal evolution of fuel and alpha-particle densitu. ion and electron temperature, and poloidal and ayal (toroidal) magnetic field. Equilibrium is enforced through a radial pressure balance equation. There is a variet $y$ of transport models available including densitv - or pressure-gradient driven diffusion, as well as thermal force terms: and. it is a trivial exercise to add new transport coefficients. The code includes a 1-D neutral-hydrogers transport description, an impurity-density-specified coronal equilibrium radiation model an up-todate pellet injecticn model. a mean-field dynamo model required to permit steadr RFP equilibria. and a fusirn-priduct alpha-particle slowing-down model. RFPBI RX has been used t, examine a wide range of specific phenomena including sawtooth oscillations. oscillating-field current drive. dinamio quasj-linear effects of Alfven waves and pressure- and current-driven instabilities. pellet injection, impurity injection, radiation collapse, soft beta limits. ignition, and reactur uperation. The codv alsc, provides profiles for linear stability calculations and for self-ronsistent coupling wit It edge-plasma transpurt models. Additionallv. a separate version (RFPELCE) treats a crlindrical 


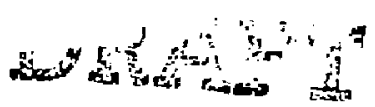

annulus and include axial loss terns for simulating edge plasmas. Another version (ZAP) treats a cylindrical annulus with a moving inner boundary of solid hydrogen, also including the external circuit equations, for simulating HDZP start-up.

A vailability Recent versions are available on the MFECC Network, contact $K$. Werley or $R$. Sebel at Los Alamos.

Users K.A. Werley, R.A. Nebel (LANL), R. Veerasingam (Penn State), R. Scardovelli (Miendelstein), J. De leaux (Phillips Petroleum)

Documentation Older versions of the code are documented through a series of $\mathrm{L}$. of Illinois reports and theses, contact K. Werley or R. Nebel at Los Alamos.

\subsubsection{SNAP}

\section{Description}

\section{Special Features}

- For rapid analysis of experimental data

- Time-independent, shifted circles

- Inpur iscludes measured profiles, radiation and neutron emission, ...

- Output includes confinement times, power balance, and profiles not measured

- 125 SNAP runs available in Texas data base

Documentation See Ref. ?

\section{8.i.1. TR.ACK}

Description TKACK is a suite of routines for computing the intersections of a trajectory with a set of nested flux surfaces, finding magnetic field components at a given point, and transforming between flux coordinates and Cartesian or cvlindrical coordinates of toroidal plasmas.

Special Features The TRACh routines are applicable to any toroidal device (axisymetric or non-axisvmnetric plasmas) and contains several ent ry points for different problems. The gecunetrv and magnetic field must be represented in spectral form (i.e.. sine and cosine expansions of $h, Z$. and $\mathbf{B}$ )

Integration with Oitser Codes TRACK requires spectral coefficients as input -thess mav be provided by 1MOMs (Sec. 6.2.9) or VMEC (Sec. 6.2.8) equilibria or by simple approximation. It has been integrated into the HIJ'ST (Sec. 81 16) and PROCTK (Ser. ??) Iranspurt codes to provide chordal informarion for neutral beam and pellet injection. and interpretation of churdal density and charge exchan se sigrals, etc. 


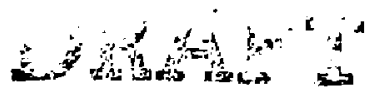

A vailability A stand-alone version of TRACK demonstrates some of its capabilities and is atailable in either $\mathrm{VAX}$ or Cray forms. The stand-alone version uses DISSPLA graphics. Contacts at ORXL (FTS prefix 624): Stan Attenberger (615)-574-0613, N.IFECC user 21070; Wayne Howberg (615)-574-1350, NMFECC user 2357.

Users ORNL: S.E. Attenberger, L.D. Horton. W.A. Houlberg, H.C. Howe.

Documentation See Ref. 2.

\subsubsection{THEORY}

Description THEORY computes the microinstability-theory-based transport coefficients given in Ref. 113. Transport from the following modes is included:

- Drift waves, including trapped electron and $\eta_{i}$

- Rippling

- Resistive ballooning

- kinetic ballooning

- high frequency $\left(\eta_{e}\right)$

Special Features This code is written to be a subroutine within the BALDLR code (Sec. 8.1.1), but can also be used as a stand-alone code to calculate fluxes from various transport mechanisms.

Availability The subroutine can be obtained on the XMFECC by typing:

cfs

get /004121/bald88/ubalon 1

end

This produces a lib file, which contains the required codes.

Users Singer ( $\mathrm{L}:$. Dt.)

Ducumentation See Ref. 31.

\subsection{J4. TRANSP}

Description TRANSP is a time-dependent. $1 \frac{1}{2}-\mathrm{D}$ transport analysis code. The data inputs to the code include plasma current $I_{p}$; surface voltage, $\mathrm{I}$, and spectroscopic measurements of hydrogen and impurity confinement times $\left(\tau_{p}^{H}\right.$, and $\left.\tau_{p}^{O}\right)$ in addition 10 profile measurements of electron. density $n_{e}(r, t)$. and electron temperature, $T_{e}(r, t)$, jon temperature, $T_{i}(r, t)$, and the radiat ed power, $P_{\text {red }}(r . t)$. On the basis of the measurements, the code solves the magnetic field diffusion equation for the poloidal magnetic field. $B_{\theta}(r, t)$ in urder to evaluate $Z_{\text {eff }}(t)$, and the current densit $y, j(r, t) 38$.

Users M. Zarnst orff (PPPL) 
Ductumentation See Ref. 38 .

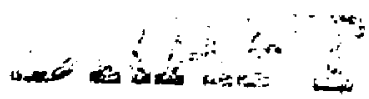

\subsubsection{TSC}

Description TSC is the Tokamak Simulation Code developed at PPPL and used extensively in Princeton and throughout the world. It can model the evolution of a free-boundary axisymmetric tokamak plasma on several different time scales. The plasma equilibrium and field evolution equations are solved on a 2D Cartesian grid, while the surface averaged transport equations for the pressures and densities are solved in magnetic flux coordinates. An arbitrary transport model can be used, but the Coppi-Tang model is used most frequently. Neo-classical resistivity, bootstrap current, auxiliary heating, current drive, alpha-heating, radiation, pellet injection, sawtooth, and ballooning mode transport models are all included. As an option, circuit equations are solved for all the poloidal field coil sy'stems with the effects of induced curtents in passive conductors included. Realistic feedback systems can be defined to control the time evolution of the plasma current, position, and shape. Required voltages for each coil system can be output as part of a calculation. Vertical stability and contral can be studied, and a disrupting plasma can be modeled.

Special Features TSC can be run in a "data comparison" mode in which case it reads specially" prepared data fles for the PBX-M, TFTR. or DIII-D experiments. For each of these, a special post-processor exists, which directly compares TSC predictions with both magnetics and kinetics dat a for particular shots from these experiments. In all modes, TSC calculates the ballooning mode stability criteria internally, and also writes files that are read by the PEST code to calculate low-n mode idual and resistive stability.

Availability The FORTRAN source file is called TSCPLB7 and is stored in CFS FILEM directory 431.PLBLIC It compiles under CIVIC, CFT $i \pi$, or CFT2, uses TV8ULIB and $\mathrm{XAG}$, and runs only on the BMA and FMA. Versions are avalable in CRPP/EPFL (Switzerland), IPP (Germany), and J.AERI (Japan). Contact S. Jardin (PPPL).

Users T. Kaiser, D. Shumaker (LLXL): S. Jardin, N. Pompnrey, C. Kessel, D. Ward (PPPL): R. Sayer. J. Whitson (ORNL); K...i. Ling, C. Bathke (LANL); J. Ramos, T. Yang (MIT): B. Merrill (EG\&G): M. Shoucri (IREQ, Canada); R. Weiner (IPP, Garching); F. Marcus (CRPP'EPFL. Switzerland): Y' MLdzuno (IPP, Xagoya): T. Tsunematsu, Y. Xakamura, Tsutsui (J.AERI, Japan).

Documentation A 58 -page users manual is avajlable from either Jardin at PPPL. The computational model is described in Ref. $5 i$, and benchmarking of the code against experiment in Rer. 6ı.

\subsubsection{IIHIST}

Description 11HIST, (Wayne Hodberg Implicit Space Time), is a time-dependent. I 1, 2-D transport code. Many parts of the code ase able 10 accommodate stellerator geometry, and work is in progress to inake the code fully compatible with both stellerators and cokanaks. The code has been written with the intent of making it available to all interested persons. It is expected that users will have some familiarity with transport theory. Documentation is primarily intemal (FORTRAX comments). Typical cases take a minute or two of Cray execution time. 


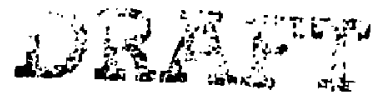

Special Features Some of the modules of WHIST are available as free-standing codes with example input and out put files. These include a pellet ablation package, the VMOMS equilihrium solver (Sec. 6.2.9), a general purpose geometry package (TRACK, Sec. 8.1.12), and a neutral beam package. These packages and the complete WHIST code are fully supported: i.e., help with their use and fixing of bugs is provided by the code developers.

A vailability An automated procedure exists to generate lax or Cray code for the WHIST code or any of its modules. The code is easily adapted to IBM or other computers. Contact the cade developers below for a version adapted to your needs.

\section{Users W.A. Houlberg, S.E. Attenberger (ORNL)}

Documentation The WHIST code is documented with comment lines within the source.

\subsubsection{ZERO}

Description ZERO is primarily a OD transport code for fitting confinement-time models to data and extrapolating to ignition. Lising prescribed parabolic profiles or density and temperature sources for ohmic heating, bremstrabhlung, and impurity radiation, alpha fusion power are readily calculated. These global power sources and sinks are balanced against semi-empirical theoretical tnodels for transport power losses from the trapped electron and ion-temperature gradient modes. The code can be run in a two-temperature, time-dependent mode to fit the models to data on confinement time. The code can run scans over current and density or take input from large-global, confinement-time data bases. The fitted power losses are then used to generate density-temperature power contours to ignition (POPCON Sec, 5.1 .2 plots). Options permit the use of Kaye-Goldston empirical models as well.

The code also contains an option for 1D one-temperature steady-state calculations with fixed density profiles and prescribed theoretical models for heat diffusivity Model auxiliary heating profules are prescribed but ohmic heating is self consistently calculated with Spitzer's law. The code operates by "shuoting" from the outer radjus to the $q=1$ surface where a sawteeth short circuit is prescribed. This is followed by interation to obtain a current profile consistent with Spitzer's lau. The code is stable to operation near marginal stability for temperature gradient mode thresholds. It is 10-30 times more efficient than the standard 1D time-dependent codes for this imited class of problems.

\section{Availability Contact R.E. Maltz at General Atomics (GA)}

Users R.E. Nalt 2 and K.R. Dominguez (G.A)

Documentation There is no detajled documentation of this code: however. a publication that has used this code can in found in Ref. 132.

\subsubsection{CROSS-REFERESCE}

Other codes that are useful for studying cross-field transport are found in Sections 11 (1.2.1 and 11.2.2. 


\subsection{2-D Transport}

\subsection{1. $\quad B 2$}

Description $B 2$ is a multi-fluid code for study of the two-dimensional structure of ise tokamak edge plasma. The code models an electrically neutral and current-free plasma containing several ion species and electrons. Each ion fluid is governed by a Navier-Stokes system of equations. Coupling between the plasma specjes occurs through ionization and recombination processes, interspecies friction, electric and thermal forces, and temperature equilibration. Parallel transport is assumed classical, while radial transport is anomalous. A simple neutral gas model is included in $\mathrm{B} 2$.

Special Features The code consists of a large number of files on the VAX, which are concatenated and shipped to the Cray for compilation and loading. Several of the files are problem-dependent routines: sample problem setups are available for ASDEX, DIII-D, JET, NET, INTOR and ITER.

A vailability Source files and the tools necessary to assemble the code are stored in CFS under user number 30137 (Rensink). Also, the code author (Bas Braams) can be reached on the MFEXET with the name BBR.AA.IS@PPC.MIFENET and has been very willing to help new users.

Users M.E. Rensink (LL.NL), B. Braans (PPPL)

Documentation The most complete code documentation is in Ref. 11. Copies may be available from the author (temporarily at PPPL). Several publications referenced in Rer. 11 describe specific applications of the code.

\subsubsection{PLANET}

\section{Special Features}

- 2-D transport in divertor and scrape-off laver

- Advective transport due to drift effects

- Realistic geometry

- Cour 'ed to DEGAS

\subsection{Parallel Transport}

\subsubsection{PHLOW}

Description PHLOW' is a time-dependent, one-dimensional code for ion transport along a single magnetic flux iube. The equations used are essentially the double-adiabatic equations of (hew. Goldberger, and Low but collision colupling between the perpendicular and parallel temperatures is used 107 . The ion equations are advanced using an explicit two-step Lax-Wendroff scheme. Either open-end or perıodic boundary conditions can be used. The electrons are represented by a pressure balance equation allowing anisotropic pressure. The electron temperature profiles can be specufied or calculated using a Spitzer thermal conduction model that is finite-differenced implicitlv. 


\section{Special Features}

- PHLOW can be coupled to the Monte Carlo code MCPAT (Ser. 7.2.2) such that MCPAT calculates the electron profiles and PHLOW calculates the ion profiles. PHLOW can also be used to give initial profiles for ion Monte Carlo calculations.

- Code output is in the form of an FR80 plot file showing profiles of ion densivi drift relocity; perpendicular pressure, and parallel pressure. Magnetic field profiles can be read from EFFI output (Sec 3.3.1) or specified using cubic splines.

\section{Availability Contact AognlieneLLL.MFECC}

Users T. D. Ragnilen (LL.NL)

Code Benchmarking The code reproduces analytic stagnant flow solutjons for the stmmetric penetration of warm plasma into a mirror cell. It also shows the expected transition tr. supersonic flow at the mirror throat for the asymmetric, finite fiow selocity case.

Documentation See Ref. 107.

\subsubsection{ZCODE}

Description ZCODE cont ains a 1-D (parallel to the nagnetic field) steady-state, fluid description of jon density ion momentum, and ion and electron temperature. The transport model assumes classical parallel conductivities with free streaming heat flux limits enforced on both the jrons and the electrons, and applies sheath boundary conditions at one side. A 1-D neutral-hydrogen transport description is included, as well as flux-surface expansion effects. ZCODE is used to help simulate edge-plasma conditions.

Special Features ZCODE is set up to interface directly with a series of other codes useful for describing edge conditions, including a radial cross-field transport code, RFPEDGE: a 2-D magnetic equilibrium code geometry from either XEQ (Sec. 6.2.7) or TORSIDO: a divertor-plate locator and shape-optimizer code. DIYLOC; and finally; a 2+D neutral-atom transport cide. DEGAS (S.c. 9.4.1).

A vailability The cude is arailable on the MFECC Network contart $k$. 1 . Werles:

Users K.A. Werley (LANL)

Documentation Documentation consists of a view'gragh package, crinlact $k$. Werley:

\subsubsection{C'ROSS-REFERENCE}

Other codes that are useful for studying parallel transport are fround in Sections 7.2 .2 and 73.5 . 


\subsection{Impurity- Transport}

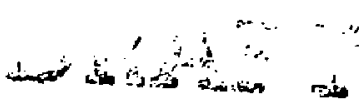

\subsubsection{MIIST}

Description .MIST is a time-dependent, I-D multi-species impurity transport code that solves the coupled continuity equations for each charge state of a given impurity element in cylindriral geometry. The radial impurity ion transport particle flux is taken together with source and sink terms. and the atomic processes of jonization and recombination to determine the evolution of the radial distribution of each impurity jon charge state in the plasma, along with other associated quantities.

Special Features MIST takes as input either fixed or time-dependent profiles of the plasma electron temperature, electron density, neutral hydrogen density and energy, etc, along with choices for the impurity transport coefficients and atomic physics to be used for a particular run. Complete (though approximate) atomic rate data is presently available in MIST to allow calculations for any impurity element, with plans to include ALADDIN (Sec. 3.1.1) atomic physics database interface capability 10 access LAEA standardized evaluated atomic data as it becomes available. The code uses an implicit finite-difference numerical scheme, which is embedded in a predictor-corrector loop to handle nonlinear transport coefficients, edge sources with finite "tecycling" coefficients. etc. Dyramic time-step control allows the calculation to adapt to changing time scales in the solutions. Among the advantages of the implicjt numerical method is that it allows transport equilibrium (time-independent) solutions to be obtained directly (for linear transport models). Such equilibrium solutions can be obtained via a single input switch setting.

IDST output is available in both tabular as well as plotted form. The tabular output can be read by various databases, post-processors. pasted into Macintosh graphics and spreadsheet programs, etc., for flexible manipulation and plotting of results in customized applications. MIST versiuns are available for the DEC VAX as well as MFECC's Cray computers. The MAX version dues not generate plotted output.

IUST code development has been primarily oriented towards providing support for experimental data analysis and modeling, with straightforward setups for these applications and out put emphasizing experimentally relevant quantities such as spectral line brightnesses, radiated power profites. and $Z_{\text {eff }}$ profiles. Transport equilibrium rurs are rnutinely used for basic interpretation of

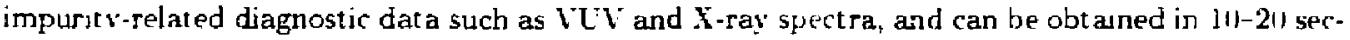
onds of $\mathrm{VAX}-8600$ time. Fime-dependent runs are used primarily for analysis of impurity injection experiments and interpretation of shots with strongly time-varying plasma profiles. Such cases are usually run using the faster original MFEC."s Cray implement ation of the code, and rypucally take 1-3 mutes on Cray-1.

\section{A valiability Contaci Russell Hulse (PPPL. 1609)2-33-2621, email HCLSE}

Lisers PPPL/TFTh (K. Hulse. B. Stratton et al), GA/DIII-D (M1.\&. Mahdavi X Bronks. pt

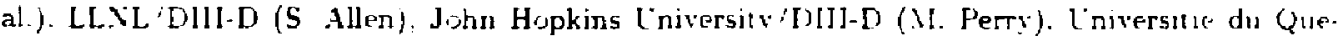
bee Tikamak de larennes ( $K$ Parbhakar. $K$ Dinoff. et al.). LASL/ZT-4U(T. Cayton). JAEK) JF] $2 \mathrm{~N}$ (H. Ogaxa)

Documentation The basic published MIST reference is hef 49 . The ISofe preprieressir usred for IIST code generation (R. Hulse, author) automatically generates dicumentaliull corl comumun 
block and input variables, and this documentation is provided for user reference along with sample input files, etc.

\section{Sources and Sinks}

\subsection{Electron-Crclotron Frequency}

\subsubsection{TORCH}

Description Ihe TORCH, (Toroidal Ray-tracing Current Drive and Heating), code traces rays that represent a microwave beam in the electron-cyclotron frequency range. Equilibrium quantities $\left(\bar{B}, n_{e}\right.$, and $\left.T_{e}\right)$ can be obtained from analytic models or from MHD codes via an EQDSK file. TORCH calculates radial profiles of power deposited and current driven. The code can also calc ulate profiles of power deposition on a calorimeter. Ray's can be reflected by mirrors in the region exterior to the plasma. TORCH can also simulate a cyclotron-emission diagnostic by computing the power emitted and reabsorbed along rays that are accepted by the diagnostic.

Special Features TORCH is organized as a set of packages that are configured by the BASIS system (see Sec. 2.1). The B.ASIS interpreter handles all input and output and provides access to internal documentation about the packages and variables.

Availability TORCH can be obtained on the NMFECC Cray-2's in a lib file that cont ains sample input files. Type cfs get /3030/totch2/torch.

Users G. Smith (LL.NL)

\section{Documentation}

\section{J.2. TORAS}

Description TOKAY is primarily a 3-D, time-independent, ray-tracing code for electron-cyclotron waves, and computes single-pass absorption rates, current-drive efficiencies, energ $v$-deposition profiles, and driven-current profiles. By interfacing to ONETWO (Sec. 8.1.9). TORAY can be used for time-dependent problems. There are many options to run the code, such as a noncircular cross-sertion with numerically given density and cemperature profiles. a circular cross-section with prescribed parabolic profiles, etc. The code uses the cold-plasma dispersion relation to trace ravs, but uses solutions of a relativistic dispersion relation for absorption. and includes a relativistic current-drive theorr.

The main frame of TORAY was developed by Amold hiritz (Hunter College) and Gary h. Smith (LL.NL). At General Atomics, the code has been improved bv installing a comput ation of relativist ic atsorption that includes a capability to model non-Maxwellian electrons by a twistemperature distribution. and also improved in the efficiency by a factor $11,-40$.

Availability Cintact $K$ Matsuda at General Atrmics (GA)

Users K. Matsuda. I. Chan. K.H. Harvey (GA), H. James (LLXL, G.A) 
Documentation There is no detailed documentation for this code: however. one publication that has used the code can be found in Ref. 89.

\title{
9.2. Ion-Cyclotron Frequency-
}

\subsubsection{A.TTENA}

Description ANTEXA (Mcley Code) calculates the time-harmonic self-consistent plasma fields from a variety of antenna types, to an infinitely long cylindrical plasma within a cylindrical conducting boundary. Single antennas or phased arrays can be handled. Antenna types include full and partial turn loops, Nagoya type III (Kharkov), rectangular apertures, saddle coils, and line currents. Radial profiles of density and temperature are approximated by steps for Gaussian, parabolic or arbitrary user input profiles. The warm plasma dispersion relation is used to compute Landau and cyclotron absorption, with a Krook operator to simulate collisional absorption. It has been particularly useful for calculating the loading resistance, and studying ponderomotive stabilization effects.

Special Features The following parameters can be scanned: radius, azimuthal angle, axial position. frequency. density. magnetic field, temperature, and parallel wave number. A second sran can be nested in the first.

A vailability ANTEXA can be abtained on the NMFECC's Cravs. Type filem rds 1437 . icrf xantenc4 xplota4. XANTEYC4 is the executable file, and XPLOTA4 is the plot package. The file ANT NEW'S contains information on the latest updates to the code. ANTENA is also available as part of the BASIS package.

Lisers G. Smith (LLNL)

\section{Documentation}

\subsection{2 $\mathrm{KCRH}$}

Description See D. Bachelor!

\section{Special Features}

Code Benchmarking

\author{
A vailability
}

Üsers

Documentation See Ref. ? 


\subsubsection{FIRC}

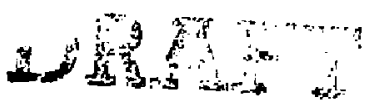

Description FIRC is a 1-D Fundanental Ion Resonance Code (FIRC). Propagation, transmission, reflection, absorption of i.e magnetosonic mode across the fundamental ion cyclotron resonance layer are computed. Solution of the three basic scattering problems are obtained: (1) Fast-mode incidence from high-field side, (2) Fast-mode incjdence from low field side, and (3) Slow mode incidence. The component of $\mathrm{E}$ along equilibrium $\mathrm{m}$. gnetic field is ignored. Warm plasma electric current is derived from linearized llasov equation within a boundary laver near the res $\mathrm{s}$. nance surface.

Special Feautres ODE solver DVERK is used from IMSL package to construct linearly independent solutions of the field equation. Appropriate linear combinations of these solutions are matched to NKB solutions vielding full wave solutions. Asymptotically growing solutions are suppressed by reduction of $r$, der method.

Availability Cont act Kava lmre or Harold Weitzner, Courant Institute of Mathematical Sciences. New York Iniversity, New York, XY 10012.

Documentation See Refs. 50.50 . and 58 .

\subsubsection{MFIKC}

Description MFIRC is a 1-[) Minority Fundamental Ion Resonance Code with poioidal field We allow the poloidal component of the equilibrium magnetic field to be non-zerc and show that the field equal icins become an intergrodifferential ststem. We compute the propagation, transmission. reflection. absorpticon of the incident fast mode across the fundamental ion cyclotron resonance laver. Silutions if the basic scattering problems are obtained: (1) Fast-mode incidence from highfield side and (2) Fast-Mode incidence from low-field side. Wam plasma elertric current is dericd from the linearized Vlasov equation within a boundary laver near the resonance surface

Special Features The int egral operat or is truncat ed and discretized. We obtain binearly-independent sijutions for this system. Appropriate lineas combinations of these solutions are matched to WhB s.jutions vielding full wave sijutions.

Availability Contact Kava Imre. Harold Weitzner. and D.C. Stevens. Crurant lnstitute of .lath-

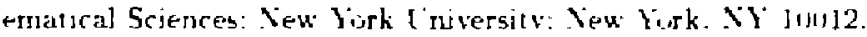

Docurnentatiou See Kefs. 134,53 . and 59

\subsubsection{SHIRC.}

Description SHIRC is a Second Harmenic Ion Resonance Cude with putoidal firld llo allew the polosilal compenent of the equlibrum magnetic field to be non zeres and show that the fietd equaturns become is fouth-urder antergrtdifferential system. We compute the prepragatin. mode conversion. transmssion. reflection. arsd absorption of the incident waves arruss the serind har-

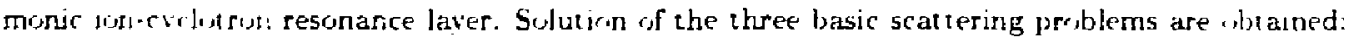




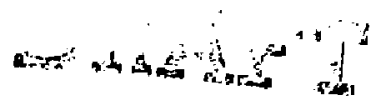

(1) Fast-mode incidence from high-field side, (2) Fast-mode incidence from low-field side. (3) Slciwmode incidence. Warm plasma electric current is derived from linearized Vlasov equation within a boundary layer near the resonance surface, as a linear functional of the equilibrium magnetıc field.

Special Feautres. We obtain the solutions of the integrodifferential system by construeting an appropriate right-hand side for the ODE corresponding to the perpendicularly stratified case. The integral operator is truncated and discretized to obtain this right-hand stze. We obtain linearly independent solutions for this system. Appropriate linear combinations of these solutions are matched $t o$ WKB solutions yielding full wave solutions.

A vailability Contact Kała Imre. Harold Wejtzner, and D.C. Stevens: Courant Institute of Mathematical Sciences: New York University: Sew York, NY 10012.

Documentation See Refs. 134, 54, and 59.

\subsubsection{IIHRC}

Description IIHRC is a 1-D Ion-Ion Hybrid Resonance Code. Mode conversion, transmission. reflection, absorption of the electromagnetic and Bernstein modes are calculated across the ioncyclotron resonance layter whese there are two resonant jon species. The majority ispecjes is at second harmonic and the minority species is at fundamental resonance on the same resonance surface. Sulutions of the three basic scattering problems are obtained: (1) Fast-mode incidence from high-field side, (2) Fast-Mode incidence from low-field side. (3) Bernstein-mode incidence. The component of $\mathrm{E}$ along equilibrium magnetic field is ignored. Warm plasma electric current is derived from the linearized Vlasov equation within a boundary layer near the resonance surface.

Special Features ODE solver DVERK is used from IMISL package to construct lineariv independent solutions of the field equation. Appropriate ljnear combinations of these solutions are matched $t, \mathrm{HhB}$ solutions yelding full wave solutions. We use reduction of order method tw eliminate growing solutions.

A vailability Contact haya Imre and Harold Weitzner. Courant Institute of Nathematical Scienres. New York [-niversity. Yew York, NY loul2.

Durumentation See Refs. 52. 55. and 58.

\subsection{THIKC}

Description THIKC is a 1-T. Third Harmonic Ion Resonance Code. Mude conversion. transmus sjon. reHertion. absorption of the electromagnets and Bernstent modes across the third harmonc ion-crclutron resonance laver are computed. Silutions of the three basic scattering probiems are ubtaned: (1) Fast-mode incidence from high-field side. (2) Fast-lude inaderse frim low-field sude. and (3) Bernstein-mode incidence. Component of $E$ along equijibrium magnetir field is ignured Hars: plasma electric cursent is derived from the linearized Vlassv equatum withn a bursdars layer near the resonance surface. 


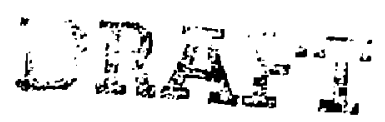

Special Features ODE solver DVERK is used from LISL package to cronstruct linearlv independent solutions of the field equation. Appropriate linear combinations of these sulutions are matched to WKB solutions yielding full wave solutions. Double reduction of order technique is used to suppress growing solutions.

Availability Contact Kaya Imre and Harold Weitzner, Courant Institute of Mathematical Sciences. New York Liuversity, New York. XY 10012.

Documentation See Refs. 51, 55, and 58.

\subsubsection{GARFIELD}

Description Grumman Aerospace RF fIELD code (GARFIELD) is designed to calculate the three-dimensional structure of ICRF fields in axisymmetric mirrors. The code solves the electromagnetic wave equation for the electric field using a cold plasma dispersion :efation with a small collision -erm to simulate absorption. The full wave solution including $\vec{E} \cdot \vec{B}$ is computed. The fields are Fourier analyzed in the poloidal direction and solved on a grid in the axial and radial di. rections. A two-dimensional equilibrium can be used as the source of equilibrium data. ICRF wave propagation and absorptior in mirrors thus includes the effect of axial variation of the magnetic field and density ' lu2.

Users G. Smith (LLNI)

Documentation See Ref. 102 .

\subsection{Veutral Beams}

\subsubsection{TFFEIA}

Description NFREYA is a Munte Carlo cude that computes the fast im birth deposition for neutral beam injertion into tokamaks and stellarators.

Special Features NFREYA carefully treats the geometry of the racuum vessel and includes thearn dwergence. focussing and aperture losses. Buture averaging over the initiai in writs is upt innal. The magnetic fields are obtained from 2-D (twkanak) or 3-D (stellaratior) cudes. The bearn geometry is mordeled in detail.

Integration with Other Codes XFREYA exists as a st and-alone cude. It has been inc,rpi, rated as a module rof the nonlinear multispecies Fukker-Planck code CQL (:QL3D (Sec 111.2.1).

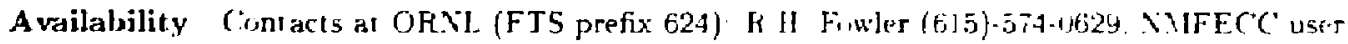

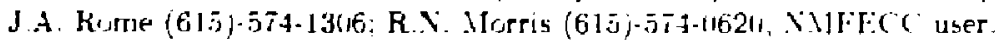

Users OKN1. K.H. Fouler. J.A. Kome K.X M.rris. I.1.YL.: (i.D Kerbe]

Dorumentation See Hefs. 29,35. 


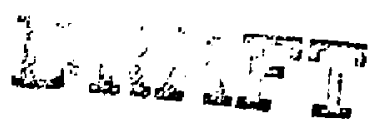

\subsubsection{BEAM}

Description BEAM is a code that computes the profile of birth points for neutral beam depasition, using a multiple beamlet formulation that does not include divergence or focussing effects.

Special Features The BEAM code is applicable to any toroidal device (axismmetric or nonaxismmetric plasmas), and includes arbitrary bearn orientation with respect to the plasma. It calculates the flux surface average of the birth current profile. $\langle v \cdot B\rangle$, as well as th: usual particle source profile $\mathrm{H}(r)$. The toroidal momentum source could easily be added as a calculation that parallels the birth current calculation. Options include circular or rectangular beam cross-sections.

Integration with Other Codes BEAM requires auxiljary routines for geometry and crosssections: and the output must be processed by a Fokker-Planck code to obtain thermal electron and ion heating and current source profiles. BEAMI has been integrated into the WHIST transport code (Sec. (1.16). In this iriplementation, BEAM uses the TRACK code (Sec. 8.1.12) for the plasma and beamlet geometry, and the MIRTH (Sec. 8.1.8) code for the themalization of the fast ions. The driven current is computed using expressions from Hirshmen and Sigmar in an auxiliary routine

A vailability A stand-alone version of BEAM that computes particle and current birth profiles and includes coupling to the TRACK routines is available in either VAX or Cray forms. Any other auxiliary routines, as implemented in the WHIST code, are also available. The stand-alone version uses DISSPLA graphics. Contacts at OR.NL (FTS prefix 624): Stan Attenberger (615)-574-0613. XMFECC user 21070: Wayne Houberg (615)-5i4-135\%. XMFECC user $23 \overline{7} \mathrm{r}$.

Users ORNL: 5. E. Attenberger, II A Houlberg.

Documentation See Refs. 3.42 .

\subsection{Seutral Particle Transport}

\subsubsection{DEG.AS}

Description The DEGAS cude solves the linearized Boltzmann equation for neutral particle transport in a fixed plasma. The solucion is obtained via a Monte Carlo method employing the pseudu-crildisjun adgotitun (PCA) for tracking particles. It includes all relevant neutral-plasma interactions for itydrogenic atorns and molecules, and for helium. Wall reflection and sputtering is modelled by a combination of data from experiments and from the TRJM and MARLOWE computer rodes. Liall composition can be specified from a list of materials commonly used in fusion experiments and reartor designs. Arbitrary three-dimenswnal geometric configurations can be treated. With special options available for rylindrical and toroidal symmetry. A full set of diagnost jes is avajlable from a post-processing graphics package, including 3-D and contour plots Particle, mument um. and energy fluxes to the walls are alsu computed. The defunition iff the grid. plasma. and walls fror DEGAS is time-consurning and verv problem-dependent the user shuuld urite a separate code to perform this task. 


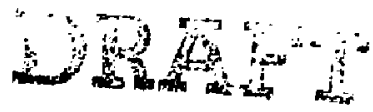

Special Features DEGAS can be run on the CRAY-2 in a multi-tasking mode. The Monte Carlo algorithm includes particle splitting and Russian roulette options to more effectively use computational resources.

A vailability Access to the code is described in a user's manual available on the N.MFECC's Cray' ?'.

Users T.B. Kaiser, M.E. Rensink (LLXL); L. Owen (ORXL): A. Ehrharde (PPPL)

Code Benchmarking The code has been used to model several fusion experiments. including TFTR, PDX, DIII-D and TMIX- $\mathrm{C}$. Published references to these applications are in the article by Heifetz ${ }^{\circ} 40^{\circ}$.

Documentation See Ref. 40. An unfinished draft of a user's manual is available on MFECC by typing filem rds 4575 .d.doc deguser.

\subsection{FENAT}

Description Solution of the energy dependent diffusion equation in two dimensions is formulated by multigroup approximation of the energy variable and general triangular mesh, fuite element discretization of the spatjal domain. Finite element formulation is done by Galerkin's method. Based on this formulation, a two-dimensional multigroup finite element diffusion theory code, FEXAT, has been developed for the transport of neutral atoms in fusion plasmas. FENAT solves the multigroup diffusion equat jon in X-Y cartesian and R-Z cylindrical/toroidal geometries. Use of the finite element method allows solution of problems in which the plasma cross-section has an arbitrary shape. The accuracy of FEXAT has been verified by comparing results to those cbtained using the two-dimensional discrete ordinate transport theory code. DOT-4.3 $36^{\circ}$.

Users T. Kaiser (LL.YL)

Documentation See Refs. 36.37.

\subsubsection{PELLET}

Description PELIET is a code that computes the mass deposition of frozen hydrogenic pellets in magnetically confined toroidal plasmas.

Sperial Features The PELLET code calculates ablation from plasma electrons and fast ions using the neutral shielding model. Additional shielding is included from the cold ablatant plasma flowing along the magnetic field. The energy distribution of incident electrons and fast ions (from) neutral beam injection or fusion alphas) is handled with a multiple energy group formalism. Several options on detail of the model are included. The geometrv is applicable to any toroidal device (axisumnet rir or non-axisymmet ric plasmas). and includes arbitrary pellet orientation uith respert to the plasma. The flux surfare average of the particle source profile is calculated 


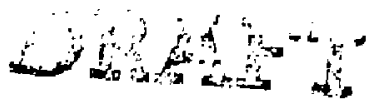

Integration with $O$ ther Codes PELLET requires auxiliary routines for the plasma geome. try. PELLET has been integrated into many transport codes (BALDLR (Sec. 8.1.1), PHOCTR (Sec. ??), TRANSP (Sec. 8.1.14), TSC (Sec. 8.1.15), WHIST (Sec. 8.1.16), plus several outside the C.S.). The TRACK code can be used to provide the required geometric information.

A vailability A stand-alone version of PELLET that computes the particle source profile and includes coupling to the TFACK (Sec. 8.1.12) routines is available in elther VAX or Cray forms. The stand-alone version uses DISSPLA graphics. Contacts at OHNL (FTS prefix 624); Stan Attenberget (615)-574-0613, NMFECC user 21070; Wayne Houlberg (615)-574-1350, NMFECC user 2357.

Users ORNL: S.E. Attenberger, M.J. Gouge, W. Houlberg, H.C. Howe. PPPL: G. Bateman, D.R. Mikkelsen, D.K. Owens, M. H. Redi.

Documentation See R.f. 46.

\subsection{Impurity Radjation Codes}

9.6. Cyclotson Emission Codes

\subsubsection{CYTRA.V}

Description CYIR.A. is a code that computes synchrotron radiation transport (enission and reabsorption), using a cylindrical approximation for the plasma.

Special Features The CYTRAN code approximates non-circular plasmas by a cylinder of equal cross-sectional area. Diffuse reflection from the walls is assumed. The plasma is divided in optically thin and thick regions. In the presence of reflection the plasma appears thick at frequencies range where most of the energe is carried, even though it is optically thin. Net flux surface-averaged. power emission/adsorption profiles are obtained.

Integra ion with Other Codes CYTRAX is run as a module in the WHIST transport code (Sec. R.1.16).

A vailability Written by S. Tamor. Contacts at ORNL (FTS prefix 624): Stan Attenberger (615)-574-1613, N.IFECC user 21(17(1: Wayne Houlberg (615)-5i-1350. NIFFEC user 2357

Users OHNL: 5 E. Attenberget W. A. Houberg

Documentation Sep Refs 122.121

\subsubsection{HOR.ACE}

Description HOR.ACE was urituen by Brb Harvey (GA) and Martin O'Brien (Culham) and incorporates Kav tracing and Absurption to calculate the net cvcloton emission tos a detertor. It calculates the power per unit frequenc' ruserved by an antenna. along nrays ravs. for a specised frequency range, and for a specified range of cvelotron hamonics. The specific untensulv 


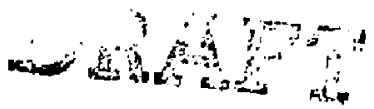

(ergs/sec) $/\left(\mathrm{cm}^{2}\right.$-steradiar-radian frequency) along each ray is weighted by wt $\left.i: i=1, n r a y s\right)$. the product of the solid angle element and antenna gain factor, appropriate to the ray. Multiplying further by the antenna area gives the power per radian frequency into the antenna.

Availability Contact C. Lasnier 2 LLNL. MFENET.

Users C. Lasnier (LL.NL)

Documentation See Ref. 9.

\subsubsection{OPAKE2}

Description OPAKE2 is a code for calculation of the emission and absorption coefficients for cyclotron radiation in a magnetized plasma. It is not intended to be a subroutine, but is a standalone code for producing a table for permanent reference.

The user specifies a plasma temperature, a direction of propagation, the highest and lowest frequencies desired, and the number of frequency entries. The code returns absorption coefficients and emissivities vs frequency for the ordinary and extraordinary modes. A relativistic Maxwellian distribution is assumed, and the plasma modes are defined via the low density limit of the AppletonHartree dispersion relation 123 :

Special Features A number of options are arailable:

1. It will provide emission (not absorption) coefficients for a loss cone distribution.

2. The distribution ran be a drifting Maxwellian.

3. The code can seturn the rorrelation matrix for the emitted electric field components.

A vailability Complete instructions for preparation of input are contained in the program listing. The cutput generated is prefaced by an explanation of the table headings and definitions. The program can be obtained by executing: filem rds 604 opake2.

Users T. Samec (T RIV)

Documentation See Ref. 123.

9.7. Lower Hybrid

\subsubsection{I1PCP38}

Description The WPCP38 code solves the equations given by Weitzner Ref 133 for wave propagarion in a plasma based on the cold plasma model The principal use of the code is for the detailed studi of the heating of a plasma at lower hybrid frequencies.

A vailability The code is available from Donald Stevents (N'T: MFECC user 1540 ) on request. 


\section{Fokker-Planck Codes}

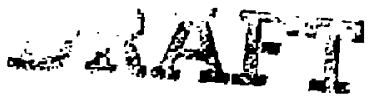

\subsection{Single FJux Tube}

\section{1.1. BAF/BACON}

Description BAV is a Bounce AVeraged 2-D Fokker-Planck code for Coulomb collisions that includes neutral beam injection, charge exchange, a simple of heating model. and finite-transit Ioss. This single-species, single region, non-relativistic code is implicitly finite differenced on a (v. $\theta$ ) velocity grid, and the resulting matrix equation solved with ICCG. The nonlinear collisional diffusion operator is determined by solving for the Rosenbluth potentials, in which a specified number of terms in a Legendre polynomial expansion are kept. The original version of the code by Cutler; et al. 20 is in non-conservation form. Subsequently, the code was rewritten so that the line density is conserved $105^{\circ}$; the name has correspondingly been modified to BACON. Even more recent Jy. L. D. Pearlstein has improved the code by using more accurate bounce-average evaluations and made the code much faster by vectorization.

\section{Sperial Features}

- The crde can optionally and reliably calculate the self-cunsistent electrostatic potential profiles along the magnetic field.

- The code has a reliable option for choosing the maximum time step.

- The code can be run in a manner to terminate upon achieving steady state.

- The code can calculate $B(s)$ including long-thin equilibrium, and has $d B / d t$ terms.

\section{A vailability Contact RogaliendLLL.MFENET}

\section{Users T. D. Rognlien (LLAL)}

Code Benchmarking BAl has been benchmarked with several other Fokker-Planck codes such as Hibrid-II (Sec. 10.1.3), S.MOKE (Se:t. 10.1.5), and CQL (Sect. 10.1.2). Also, it agrees with analytir calculations such as Pastukov confunement in mirrors.

Documentation See Refs. 20,105.

\section{1) !.2. CQL}

Description The bounce-averaged Fokker-Planck code CQL-(Collisional QuasiLinear)-has been in development for a number of years and is now available in package form. The theoretical basis for most of the physics incorporated in the CQL program is presented in the article by Kerbel and Mrcis 6.3. The code has been applied to solve a varietv of problems demonstrating its range of versatility. A few of these applications have been reported in the recent literature. includung such varied subjects as ICKF reactivity enhancement, runaway rates and resistivjty for ariblrary inverse aspect ratios. relativistic corrections 10 runawav rates and resistivitr. ECH in whamaks, the effects of collisions and finite spectral width on resonant diffusion, and neutral beam curtent drute efficiency 65 . 


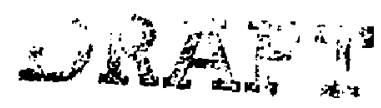

Special Features The computer code CQL is designed to solve the multi-species bounce-averaged Fokker-Planck equation complete with

- non-linear (and/or linear) collisional terms

- multj-mode RF quasilinear terms

- source terms (e.g. charge-exchange, fusion, neutral beams)

- loss terms (e.g. non-confinement boundaries, charge-exchange phenomenological models).

A vailability The source code may be accessed through FILENI in directory . . 102 of user 714. Details of what is available in this directory, and how to compile the code is sound in Refs. 65.64.

Users G. Kerbel (MFECC)

Documentation See Refs. 63,65:64.

\subsubsection{HYBRID-II}

Description The computer program HYBRID-II solves the time-dependent, two-dimensional, nonlinear Fokker-Planck equations for an arbitrary number of ion species. It makes use of the general purpose Fokker-Planck package FPPAC 123 . The two velocity dimensions are speed and pitch angle, and $i t$ is assumed that the electrons are Maxwellian.

The program may be applied to problems in the areas of both miror confinement and toroidal confinement. In the former case, a self-consistent ambipolar potential profile may be computed to assure charge neutrality, and it is also possible to model both the plugs and the sulenoid in a tandem mirror device 123 .

Availability Relevant files are located in directory public under user number 723 . File HYBRID2 is the source file. Precompile using PRECOMP with the CRAY option, compile using CFT. and Ioad TV8ULIB and OMNILIB.

Users A. Futch (LLNL), A. Mirin (MFECC)

Documentation See Refs. 123,67,96.

\subsubsection{SFP}

Description SFP is a simplified, easily modifiable code has been developed tis determine the Alfien wave of neutral beam driven toroidal plasmas :14. 


\section{Special Features}

1. Kennel-Englemann infinite medium quasilinear theory examining particle-wave power transfer for the shear, compressional and anomalous Doppler mode.

2. Linearized collision operator.

3. Expansirn in Legendre polynomials, result in a set of coupled ODE's in the speed coordinate.

4. Beam growth and electron damping terms computed for the collisional distribution.

5. Useful for parameter surveys.

6. Code driven by BASIS.

\section{A vailability Contact Campbell LLL.MFENET}

Users R. B. Campbell (LLNL)

Documentation See Ref. 14.

\subsubsection{SMOKE}

Description S.1OKE is a relativistic. bounce-zveraged, muti-region Fokker-Planck code for studying the physics of plasmas on a collisional time scale. It is capable of treating a plasma trapped in magnetic and potential wells of general shape as in a tandern mirror or a tokamak. The numerical method used is a combination of mapping tectunique and a Galerkin finite element method.

Special Features The code iscludes:

- nonlinear norrelativistic collision operator

- lineas non-relatiristic collision operator

- linear relarivistic rollision operator

- quasilinear of diffusion sperator

- synchotron radiation effects

- neutral beam injection terms

A vailability Contace Y' Matsu 3 OLLL. MFENET

Users Y. Matsuda (LLNL)

Documentation See Rer. 90. 


\subsubsection{CQL3D}

Description CQL3D is a multi-flux-surface version of the Fokker-Planck code CQL (Sec. 10.1.2). The code solves the Fokker-Planck equation simultaneously on a large number of flux surfaces. Depending on the type of problem, modules couple the various flux surfaces. For instance, for ECH a wave damping calculation computes the energy density in space (and thus the olectric field strength). and the Fokker-Planck code computes the losal power absorption. The code iterates tu steady state. For neutral beams, a Monte Carlo neutral beam injection code is used to determine the local fast ion source. An MHD equilibrium code module allows CQL3D to utilize non-circular flux surfaces. if desired.

\section{Special Feat ures}

- ron-circular cross sections

- multi-fiux surface

- ECH. Neutral Beams

- Fast-CRAY-2 only

Availability Cuntact G.D. Kerbel.

Lisers M. G. McCov and G. D. Kerbel (NMFECC). R. Harvey (GA)

Ducumentation See Ref. 91 .

\subsubsection{FPT}

Description The differential equations of plasma transport in a toroidal confinement systerr are solved. Several energetic ion species are described bv velocity space distributicn functions (depending on speed. pitch angle and distance from the magnetis axis). and their slowing down and scattering are modeled numerically by the complete noninear Fokker-Planck operatur. The plasma iuns and electrons are described by a multi-fluid transport model.

The piolodal flux is taken to be a function only of $r$ (the distance from the magnetic axis). s. that constani flux surfaces are circular tori Each plasma species is described by a separate radial

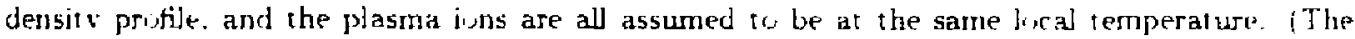
electrons have (heir oun temperature profile.) Oniy the poluidal component of the magnetic field varies with time.

Seural transport is calculated using a multispecies generalization of the Hughes. Post Misnte Carjo cude. Beam deposition is calculated using the XFREYA cude of Lister, et al 123 (Soc 9.31 ).

Avalability Rejevant files are located in directury public under uses number 723 . Fild. FI'T is the source file. HHEAMS and BNELT are binarv files, and SWLDAT is a data lile. Precimpile using PHECOMP with the C'HAY' uption. compile using CFT: and load BBE.MIS, BNEL "I. TYrill.IB. and OAISILIB. APLDAT should be is dise when exeruting 123 . 
Documentation See Refs. $123,95,66$.

\section{Stability Codes}

\subsection{Magnetohydrodynamic Stability.}

\section{J.1.1. FLOR.A}

Description FLORA solves, in a 2-D domain $(z, \psi)$, for the linearized stability of a long thin axis.mmet ric equilibrium. It uses an initial-value method in which an equilibrium is given an initial pertusbation to its magnetic $B$ field, and the time behavior of the perturbation is followed. The perturbation has been Fourier expanded in the azimuthal $(\theta)$ direction and each mode $(m)$ must be examined separately: The values of $m$ can be arbitrary with an upper limit around $O\left(10^{3}\right)$ because of accuracy consideration as the modes become more highy localized.

Users B.I. Cohen (LLNL)

Documentatiun See Rer. 31,16.

\section{G.ATO}

Description GA low'n Toroidal stability code (GATO), which is similar to PEST, but never misses an unstable mode. It uses "EQDSK" format file for input, and treats limited or diverted plasmas. with or without a flux-conserving wall.

Users T. Kaiser (LLNL), I. Lao (GA).

\section{Documentation}

\subsubsection{PEST}

Description

Special Features

Availability

Users

Documentation

111. TEARING

Description see G. Bateman! 
Special Features

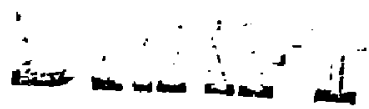

Code Benchmarking

A vailability

Users

Documentation See Fef. ?

\subsection{Resistive MHD}

\section{1i.2.I. CART}

Description CART is the 3-D nonlinear resistive MHD code, emploving a reduced set of tokamak equations with Cartesian coordinate mesh in the poloidal plane and spectral representation in the toroidal direction. This cade is particularly suited for treating up-down asymmetric noncitcular cross section with or without a magnetic separatrix, all of which characterize realistic DIII-D singlenull divertor discharges with high pressure or high current.

Past applications have been to the global mode cascade and magnetic fluctuation simulations using actual DIIl-D discharge equilibria (generated by EFIT 86,81; to the effects of magnetic separatrix and pressure pedestal on finite beta kink-tearing modes 79, and to $\mathrm{t}^{2}$. stabilization of externa] kink and axisymmetric modes in a highly elongated tokamak [78.

Possible future applications might be the transport study of multi-heilicity global ideal and resistive MHD modes especial]y with high current and/or high pressure.

Availability Contact J.K. Lee at General Atomics (GA).

Users J.K. Lee. 1.1. Lay. W. How (G.A)

Ducumentation There is no detailed documentation for this code. Some publications that have used the code jnclude Refs. 86, 81, 79, and 78 .

\subsubsection{H1B-G}

Description HIB-G is the GA version of PPPL's $3-D$ resistjue-MHD code HIB (bv W. Park) with various extensions. Being spectral both in poloidal and toroidal directions, this code has been applied to almost circular cross-section tokamaks (theoretical and D-III equilibria).

An important extension was made for simulations of 3-D MHD turbulence driven bv resistive ballooning modes ' $8 T$, adopting various pressure evolution methods to facilitate the sreadv state or saturation of these nonlocal modes.

Another extension was to the linkage to realistic experimental D-IIl equilibria 82 with minv useful code diagnostics such as the efficient calculation of various magnetic island sizes with time.

Availability Contact J.K. Lee at General Atomics (GA). 


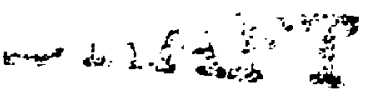

Users J.K. Lee, J.S. Kim, R.E. Waltz, M.5. Chu (G.A).

Documentation There is no detajled documentation for this code. Some publications that have used the code include Refs. 87, 82, 80, and 83.

\subsubsection{RPD}

Description RPD is a 3-D, full-resistive MHD initial value code for a poloidal divertor tokarnak with a non-diverted option available. RPD advances 3-D, full-resistive MHD equations in a fournode poloidal divertor tokamak with square cross section. The divertor separatrix and scrape off region are included as part of computational domain. The code is fully toroidal and compressible. The equations are Fourier-analyzed in the toroidal direction only and finite-differenced in two Cartesian directions of constant toroidal angle plane. A new, semi-implicit time-advancement scheme is employed for higher code efficiency. Both linear and nonlinear options are available. The number of divertor rings can be changed with moderate amounts of code modifications. The code can be extended to couple transport effects with resistive-MHD instabilities in such devices.

Code Developers Ejjiro Lchimoto (Courant Institute, lew York Lniversity) and James D. Callen (Lniversity of Wisconsin-Madison).

\section{Special Features Language: FORTAAN77. Compiler: CIVIC}

A vailability Contact Ejjro Lchimoto, Courant Institute, New York Liniversity: 251 Mercer St.. New Sork, NY 10u12; Tel. No. (212)-998-3273: MFE user No. 1556.

Users Eijiro Lchimoto and Zuoyang Chang (Lniversity of Wisconsin-Madison)

Documentation See Ref. 124.

\subsection{Microstability}

\section{ALFH'S}

Description The Alfvén-Waves code ALFWVS produces st abjlity results for Atrén-jun-cyelotron (AlC) instability in

1. a unifurm magneric field $(k=11)$

2. a mirror magnetic field $\left(k_{-}=0\right)$

3 a sharp-boundary, cylindrical, DT plasma $\left(k_{2} \neq 0\right.$ : Instability is driven by a particles)

For the first two configurations. a large variety of ion describution functions is avalable. For the thrd, the $\alpha$ dist ribution is computed.

Special Features In addition to microstability constraits, the code calculates mirrur-mude (equilibrium) limits, a wall-stabilized MHD limit, and the adiabaticity limst. 


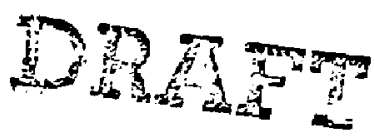

Availability Trpe filem rds 3030 .alfwvs xalfus.

\section{Users G. Smith (LLYL)}

Documentation A document is available that gives steps for running the code. descriptions of the variables, and an extensive example run (with commentary). Type filem rds 3030 . alfirvs alfivs/d. See als; Heis. I $15,117,116$.

\subsubsection{MISE:}

Description MICE code calculates microstability to jon-cylotron (and harmonics) lass-cone and anisotropy driven (not $A J C$ ) modes in mirsor geometry. The code considers. in detail the inhomgeous medium and the 3-D nature of the geometry. The formalism leads to a balloon equation which is sulved using LSODE. Hesonart denominators are treated kinetically.

Special Features The code will track marginal stability or equivalently the most unstable case.

A vailability Contact L. D. Pearlstein oLLL.MFEnET.

Users L. D. Pearlstein (LLXL)

Documentation Jone ists.

\section{I1.4. Turbulence}

\subsubsection{DIA}

Description DIA treats the dirert interaction approximation statistical closure scheme for elertrostatic electron drift-wave turbulence in sheared slab geometry. Vearly Boltzmann electrons are treated with an it model. The code solves for the correlation function of the electrostatic portential. I. in a $30 k_{z} \cdot k_{y} . k$ representation. The frequency spectrum is represented by the pole approximatiun suitable for dimination by a single branch (also called lorenta model). There are separate dinamical equations for $I$ (wave-kinetic equation) and the norlinear dispersion relation for the nonlinear frequency stuft and decorselation rate (line broadening). Thus nor inl wave-wave nunlinear coupling effects are included but also wave-particle nonlinear resunance broadening and polasizalon shieldug (Jon (ompton scat tering effect). 3D spectra for the correlation function and resonance bruademing as well as $\vec{E} \times \vec{B}$ diffusion are calculated via an iterative procedure

Availability (intact K.E. Haltz at General Atrmics (GA)

Users K.E Waltz and $\mathrm{K} \mathrm{K}$ Domunguez at Gerzeral Atomirs (Gi).

Documentation Thero is noducumentatun fro this cude: however. a publication that has used the cosde can ur frund at Hef. $12 \pi$ 


\subsubsection{DRIFT}

Description DRIFT is a 3D-electrostatic, nonlinear drift-wave code used to study drift-wave turbulence in tokamak geometry. It is a mixed space-spectral ( $r, m, n$ ) cude with global boundary conditions numericaly similar to standard nonlinear resistive MHD (Sec. 11.2) codes. It time advances the jon-continuity equation or generalized-vorticity equation to obtain the electrostatic potential perturbations. The parallel ion-motion and $\vec{E} \times \vec{B}$ convection of adiabatic ion-pressure perturbations are advanced as well. The code runs on a single or multiple helicity $m, n$ grid trs treat ion-temperat ure gradient-mode turbulonce in a sheared slab or tokamak geometry (with sheared and curved magnetic field) as well as electron drift-mode turbulence driven by a model (i $\delta$ ) nonadiabaticity for the nearly Boltzmann electrons. The background gradients are fixed and the $\vec{E} \times \vec{B}$ diffusion of density or jon pressure is computed. FLR effects are included and jon Landau damping us modeled wijth a parallel viscosity.

There is a numerically similar code TEAR ??, which treats nonjinear simulations of low'-m (MHD) tearing modes.

\section{A vailability Contact R.E. Waltz at General Atomics (G.A).}

Users R.E. Waliz.

Documentation There is no documentation for this code: however, a publication that has used the code can be found in Ref. 130 .

\section{I +.3. TWOFLD}

Description IWOFLD is a nonlinear-electomagnetic drift-wave code used for studving turbulence relevant $t$, trokamaks. Perturbations are represented on a $k_{x}$ : $k_{y}$ grid. and the effective curvature drift and paralel wave-number are taken as fixed paraneters. The code can treat a hierarchy of collisional twiofluid models staring with the two-field (TWOFLD) vorticit $y$ and electron-continuity equatiuns for the electric-field potential and density perturbations. This system describes isothermal electrustatic resistive interchange modes. Adding the jon parallel-motion equation couples the jon sisund branch and adding an explicit Ohn's law to advance the parallel magnetic-vector potential allow's electromagnetic effects including the ideal MHD (Sec. 11.2) ballooning modes. A trapped electron three-flud model abtains by the addition of a trapped electron collisional-continuty equatuon and mudifying Ohm's law to respond only to untrapped electrons. In these models. only $\vec{E} \times \vec{B}$ particle transport is significant. Further, electron-temperature perturbations can be advaneed by a collisi nal heat-conduction equation an the adiabat ic pressure convection equations. The contperature perturbations allow simulation of electric and ion heat flows while intruducing a stabllizing element to the electron-drift branch and destabilizing the ion-drift $\left(\eta_{i}\right)$ branch. The must crmplicated case involves the time advancement of sjx fields.

\section{A vailability (ontact R.E Waltz at General Atomics (G.A)}

Users R.E. Maltz

Documentation There is no detailed dorumentation for this code:however. some publications that have used the code can be fousd in Kefs. 128. 129, and 131 


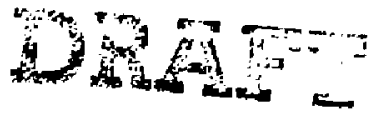

\subsection{C'ROSS-REFERENCE}

Other codes that are useful for stability analysis include Sections 8.1.15 and 8.1.15. 


\section{References}

1. S.E Altenberger, W.A. Houlberg, Nuc. Technol./Fusion, 4 Part 2, 129 (1983).

2. S.E. Attenberger, W.A. Houlberg, S.P. Hirshman, J. Comput. Phys., 72435 (1987).

3. S.E. Attenberger, W.A. Houlberg, N.A. Lckan, "Transport Analysis of Ignited and Current. Driven ITER Designs," to be published in Fusion Technol. (Code application.)

4. A.G. Arrnstrong, GFUN3D User Guide, Rutherford Laboratory RL-76029/A (1976).

5. F. Baver. O. Betancourt, and P. Garabedian, A Computational Method in Plasma Phystcs, New York, Springer-Verlag, 1978.

6. F. Bauer, O. Betancourt, and P. Garabedian, Magnetahydrodynamic Equilibrium and Stabulity of Stellarators, New York, Springer-Verlag, 1984.

-7 F. Bauer. O. Betancourt, P. Garabedian, and M. Wakatani, The BETA Equilibrium. Stabilaty and Transport Codes: Applications to the Design of Stellarators, New York. Springer-Verlag (198\%).

8 A. Barliss. L. Jbanez, and J.T. Hogan, "Implementation of Grad's Alternating Dimensions Algorithm for 3D Classical Transport," Plasma Physics, 28, 957 (19___)

9. G. Bekefi, Radiation Processes in Plasma, Wiley (1966), pp38, 48, and 315.

I0. C.K. Birdsall and A.B. Langdon, Plasma Physics va Computer Simulation, McGraw-Hill, New York, 1985.

11. B.J. Braams, A Mult-Fluid Code for Simulation of the Edge Plasma in Tokamaks, NET Report Xr. 68. Jan. $198 \overline{\text {. }}$

12 M.L. Bruhn. TADDPACK-A Computer Code Pachage for Modeling the Thermal Effects on the Divertor in Tokamaks During Plasma Disruption, Lawrence Livermore Sational Laboratory, Livermore. CA (to be published) (1987).

13 M.L. Bruhn. L.J. Perkins, Modeling of Thermal Effects on Tiber II Divertor during Plasma Disruption. (IEEE $12^{\text {th }}$ Symposium on Fusion Engineering), Lawrence Livermore Xational Laboratory. Livermure. CA, LCRL-96814 (1987).

If K.B. Camphell. G.D. Kerbel, "A Study of Alfién Wiave Driven by Neutral Beam Injection in ITEK." Laurence Livermore Natjonal Laboratory, Livermore, CA. LLNL-iter-1089-016

jj E.J. Caramana, "The Long-Time Evolution Approximation for a Quasi-One-Dimensicnal Plasma Sistem." Phys. Flurds 28, 35̄̄ (1989).

16 B.I. Cohen, et al., "Interchange, Rotational, and Ballooning Stability of Long-Thin Axisymmetric Sistems with Finite Orbit Effects". Phys. Fluzds 29, 1058 (1986).

17 B.1. Cohen, et a1., Phys. Fluids 18. 740 (1975).

'18 B.I. Cohen, et al., Lawrence Ljvermore National Laboratory, Livermore, CA, LCRL-97682.

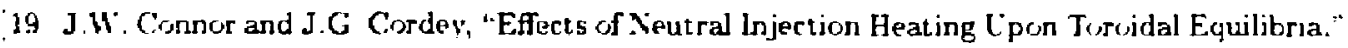
Nuclear Fusian 14, 185 (1974). 
20. T.A. Cutler. L.D Peartstein; and M.E. Rensink, Computation of the Bounce-Average Code, Lawrence Livermore National Laboratory, Livermore, CA, UCRL-52233 (197j).

21 R.S. Devoto, J.D. Hanson, SIGl-A Code to Evialuate Plasma Reaction Rates to a Specified Accuracy, Lawrence Livermore National Laboratory; Livermore, CA, LCRL-52559 (1978).

22. R.S. Devoto, K. Tani, and M. Azumi. Computation of Self-Consistent 2-D WHD with NeutralBeam and Bootstrap Currents in Elongated Plasmas, 15th Eur. Conf. on Cont. Fusion and Plasma Heating, Dubrovik (1988).

23. R.R. Domingtiez and R.E. Waltz, Nuclear Fusion 27, 65 (1987).

24. P.F. Dubois, "Writing Basis Packages," Lawrence Livermore Natjonal Laboratory, Livermore, C.A, LLNL Report W-194 (Nor. 1986).

25 P.F. Dubois and Z.C. Motteler, "Basis User's Manual," Lawrence Livermore National Laboratory: Livermore, CA, LLNL Report M-189 (Jun. 1987).

26 P.F. Dubois. Z.C. Motteler, and P.A. Willmann, "The Basis System," Chapter 6, Laurence Livermore National Labratory, Livermore, CA, LLXL Report M-225-DRAFT, p. 25 (Mar. 1987 ).

27 M.E. Fenstermacher, ML:HA $K^{-}$-A Computer Code for Modeling Plasma Power Balance and Current Drve in Tokamaks, Laurence Livermore National Laboratory, Livermore, CA. LCID$21038(1987)$.

28 J.J. Fillben. "DATAPLOT-Introduction and Overview," NBS Special Publication 567. Jun. 1984

29 R.H. Foxler. J.A. Holmes, J.A. Rome; "MFREYA-A Monte-Carlo Beam Deposition Code for Yoncircular Tokamak Plasmas:" Oak Ridge National Laboratory Report ORNL/TM-6845 $(19801)$.

3ı R.P Freis. B.1. Cohen, User's Manual for the FLOR.t Equalibrim and Stabulty Code. Lawrence Livermore National Laboratory, Livermore, CA, LCID-20400 (Apr. 1985).

31 E.S. Ghanem. C..E. Singer. L'. Ill. Fusion Studies Lab Rep.. FSL-241 (1988).

32. H. Grad and J. Hogan. Phys. Rer. Lett. 24, 1377 (1970),

33 H. Grad. F.X. Hu and [I.C. Stevens. Proc. Yat fcad. Sct. ('S.4. 72, 3789 (1975)

34 H. Grad. "Survey ol 1-1/2 D Transport Codes." Keport MIF-93, COO-3077-15t. Magnet.,flud Dynamics Divisiun, Courant Institule of Mathematical Sciences. New York University, 1978

$35 \mathrm{~J}$ II Hanson and K.S Devoto. TIBRO H: A Cade to Compute Trajectories of Charged Particles in .Magnetzc Fzelds, Lawrence Livermore National Laboratory. Ljvermore, CA. LCKL-5218T (1976).

36 .M.Z. Hasan and R.W. Conn. A Two-Dimensional Fante Element Wulligroup Diffusion Theory for Neutral Alom Tmnsport in Plasmas, CCLA/PPG-916 (Feb. 1986)

37. M.Z. Hasan and R.W. Conn, J. Comp. Phys. 71, 37! (1987). 


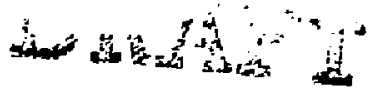

38. R.J. Hauryluk, Physics of Plasmas Close to Thermonuclear Conditions, B. Coppi, ed., Vol. 1. p. 19 (CEC, Brussels, 1980).

[39' F.J. Helton, R.L. Miller, J.M. Rawls, J. Comp. Phys. 24, 117 (1977).

40. D.B. Heifetz, Physics of Plasma-Wall Intenactions in Controlled Fusion, D.E. Post and R.Behrisch, ed. (PJenum, 1986).

41. F.L. Histon, R.D. Hazeltine, "Theory of Plasma Transport in Toroidal Confinement Systems." Rev. Mod. Phys. 48, 129 (1976).

42. S.P. Hirshman, D.J. Sigmar, Nuclear Fusion 211079 (1981).

43. S.P. Hirshman, J.C. Whitson, Phys. Fluids 26, 3553 (1983).

44. M. Hossain, M. Kress, P.JoHuPhysA Fhladk2eng573 (Arsa3)Phys. Rev. Let1. 58, 487 (1987).

45 M. Hossain, M. Kress, P.Y. Hu, A.A. Blank, and H. Grad, Phys. Fluzds 31, 2165 (1988).

16. W. Houlberg, S.L. Milora and S.E. Attenberger, Nuclear Fusion 28, 595 (1988)

47. M.-Y. Hsiao. K.A.Werley and K.-M. Ling: "A One-and-a-Quarter-Dimensional Transport Code for Field-Reversed Configuration Studies; A User's Guide for CFRX," Los Alamos National Laboratory, Report LA-11212-1IS (1988): also to appear in Computer Phys. Communications.

48. F.A. Hulse, ALADDIV-A Labeled Atcmic Data Interjace for Fusion Applications, IAEA Consultant's Meeting on the Atomic Physics Database Fusion Applications Interface. May 9-13, 1988, Henna, Austria, IAEA sumnary report INDC(NDS)-211/GA, Sep. 1988.

49. R..! Hulse. Nuclear Technalogy/Fusion 3. 259 (1983).

5u. K. Imve and H. Weitzner, Mfode Coupling Between the Fast and Slow Branches of the Extroordinary Mode at the Fundamental Ion Cyclotron Resonance. Annual Sherwood Control Fusion Therrv Conference (Penta Holel, New York) CIMS XYL New York, NY', April 14-17.

a1 K. Inre and H. Weitzner, Third Harmonic Ion Cyclotron Resonance Heating submitted to 28th Annual Meeting of Division of Plasma Phusics. American Phrsical Society. held November 37. 1986. Baltimore Convention Center. Baltimore, Maryland: Bull. Am. Phys. Soc. 31, 1421 (1986).

52. K. Imre and H. Weitzner, Ion-Ion Hybrid Cyclotron Resanance Heatung submitted to $2 \&_{1} \mathrm{~h}$ Annual Meeting of Division of Plasma Phrsics. American Physical Societv. held Xixember 3-7. 1986. Baltimore Convention Center, Baltimore, Maryland. Bull. Am. Phys. Soc. 31. 1421) $(1986)$.

53. K. Inre, D.C. Stevens, H. Weitzner, and D.B. Batchelor, Poloudal Field Effects on ICRH in Large Tokamaks. 1988 Annual Sherwood Controlled Fusion Theory Cunference (Gatlinburg. T.i). Oak Ridge X.L. April 18-20 (1988).

54. K. Imre. D.C. Stevens, H. Weitzner, and D.B. Batchelor. Polondal Field Effects in Second Harmonic ICRH, 31th Annual APS-DPP Meeting (Hollywood, FL). November (1988). Bull. Am. Phys. Soc. 33, 2019 (1988). 
55 K. Imre and H. Weitzner, "Wave Propagation Across lon Cyclotron Resonance Harmonic Layers" (accepted for publication in Plasma Phys. Contr. Fusion).

¡56j Introduction to IDL, Research Systems; Inc., 2021 Albion St., Denver, CO 80207, Feb. 1987.

[57. S.C. Jardin, X. Pomphrey, and J. Delucia, J. Comp. Phys. 66, 481 (1986).

'58. E.F. Jaeger, D.B. Batchelor, K. Imre and H. Weitzner, Plasma Heating in Stellarator/Torsatron and in Tokamaks by Ion Cyclotron Frequency Haves, Proceedings of 11 th Internationa. Conference on Plasma Physics and Controlled Nuclear Fusion, IAEA November 13-20. 1986. Kyoto, Japan, Vol. 2, p463.

59. E.F. Jaeger, D.B. Bat chelor, K. Inre, H. Weitzner, and D.C. Stevens, Full Have Hodelling of Ion Cyclotron Heating in Tokamaks (Proceedings of 12th Intemational Conterence on Plasma Physics and Controlled Nuclear Fusion, IAEA, November 13-20, 1988, Xice. France, to be published).

$61^{\circ}$ S.C. Jardin, J. Delucia, M. Okabayashi, X. Pomphrey, M. Reusch, S. Kave, and H. Takahashi, Nucleat Fusion, 27, 569 (1987).

61. T.B. Kaiser. W..M Xevins and L.D. Pearlstein. Phys. Fluzds 26, 351 (1983).

62. T.B. Kaiser and L.D. Pearlstein, Phys. Fluzds 26, 3053 (1983).

63. G. Kerbel and M. McCoy, Phys. Fluids 28, 3639 (1985).

61. G. Kerbel and M. McCoy, The Second Release of the Bounce-Averaged Fokket-Planck Code (CQL), NMFECC Buffer 12, 30 (Feb. 1988).

65 G Kerbel. M. McCoy. and M. Franz, "Preliminary Guide for the Lse of the Bounce-Averaged Fokker-Planck Cude (CQL)" NMFECC BLFFER 11, (Feb. 1987).

66 J. Killeen. et al. "A Fokker-Planck/Transport Model for Xeutral Beam Driven Plasmas," Modern Plasma Physics. p. 395, (1981). IAEA. Vienna.

67. J. Killeen, et al. "The Sulution of the Kinetic Equations for a Multispecies Plasma." Mlethods in Computational Physics 16. 389 (1976).

68 11 Kress. "Back-A veraging," J. Comp. Phys. 76, 201 (1988).

69 D. Lager and S. Azevedo. SIG, A General-Purpose Signal Processing Program. Lawtence Livemore Xational Laboratory, Livermore. CA, CCID-19912 (1985).

iil A.B Lasgdon and B.F. Lasinski. .Jeth. Comp. Phys. 16. 327 (1976).

71 L.L. Lau, Comput. Phys. Commun. 31, 201 (1984).

72 L. Lars. Documentation for the Equilibrium/Fitting Code EFIT and MFIT. available on the GA VAX system.

73. L.L. Lau, S.P. Hirshman, and K.M. Wieland, Phys. Flunds 24, 1431 (1981).

74. L.L. Las, R.M. Wieland. W. A. Houlberg, and S.P. Hirshman, Comput. Phys Commun. 27. 129 (1982) 


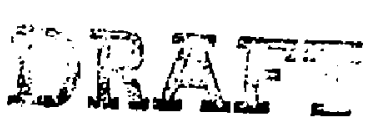

75. L.L. Lao, H.M. Mieland, W.A. Houlberg. and S.P. Hirshman, Comput. Phys. Commun. 30. 107 (i983).

¡76. W.S. Lawson, "PDW1 User's Manual," Memo LCB/ERL M84/37, ERL LC Berkeley, CA 94720 .

77. W.S. Lawson. J. Compul. Phys., in press.

78. J.K. Lee, Nuclear Fusion 26, 955 (1986).

79' J.k. Lee, Phys. Fluids, 1629 (1986).

80. J.K. Lee. Phys. Fluids 27, 772 (1984).

81. J.K. Lee, Plasma Physics and Controlled Fusion 30, 415 (1988).

82. J.K. Lee, L.C. Bernard, F.J. Helton, R.D. Stambaugh, and E.J. Strait, Plasma Physics and Contralled Fusion 28, 259 (1986).

83. J.K. Lee and AJ.S. Chu, Nuclear Fusion 24, 1360 (1984).

84'. J.K. Lee, M.S. Chu, F.J. Helton, and W. Parks, Phys. Fluids 27, 607 (1984).

85. J.K. Lee and X. Ohyabu, Phys. Fluids 27, 2874 (1984).

86. J.K. Lee, E.J. Strait, L.L. Lao, and T.S. Taylor, General Atomic, San Diego, C.A, GA-A19521 (Jan. 1989) submitted to Nuclear Fusion.

87. J K. Lee and f.E. Waltz, Bull. Am. Phys. Soc. 29, 1386 (1984).

88. "LOCLS Database Sỵstem," Rev. Sci. Instrum. 59 (8), 1780-2 (Aug. 1988).

89. K. Matsuda. IEEE Transactions on Plasma Scjence 17, 6 (1989).

91) Y'. Matsuda and J.J. Stewart, "A Relativistic Mlutiregion Bounce-Averaged Fokker-Planck Code for Mirror Plasmas," J Comp Phys 66: 197 (1986).

91 Mi. G. McCor and G.D. Kerbel, Comput. Phys. Comm. 40, 115 (1986).

92. B. Mclev. MIT report PFC/RR-84-12.

93. B. MrTev L'ser's guide for ANTENA, MIT report PFC/RR-84-13.

94 R.L. Miller. Nucl. Fusion 20, 133 (1981)).

95 A A. Mirin et al. “A Radial Transport/Fokkes-Planck Model for a Trkamak Plasma," J. Comp. Phys. 23, 23 (197\%).

9f; A.A. Mirin. Hybrid II, A Two Dimensional Multspectes Folker-Planck Computer Code. Lau'rence Livermore National Laboratory, Livermore. CA, LCHL-51615 (1975).

97. R.X. Morris. F.H. Fowler, J.A. Rome, T.J. Schlagel, Fusion Technol. 12, 281 (1987).

98 1.D. Prarlstein, et al., TEBASCO User's Guide, Law'rence Livermore lational Laboraliorv. Livermore, CA, LCID-19869 (1983). 
99 L.D. Pearlstein, W.A. Tewcomb. and T.B. Kajser, Phys. Fl. 24, 1326 (1981)

100. W. Perkins, J.C. Brown, MAFCO-A Magnetic Field Code for Handling General Current Elements in Three Dimensions, Lawrence Livermore National Laboratory, Livermore, CA, UCRL- 7744 (1966).

$101^{\circ}$ W. Pfeiffer and R.E. Stockdale, ONETHO-A Computer Code for Modeling Plasma Transport in Tokamaks, GA-A 16178 UC-20, GA Technologies Inc. (1980).

102 M.K. Phillips and A.M.M. Todd, "The Numerical Solution of ICRF Fields in Axisymmetric Mirrors:" Computer Physics Communications 40, 65 (1986).

103 R.L. Reid, ETR/ITER Systems Code, Oak Ridge National Laboratory, Oak Ridge, TN, ORNL/FEDC-87/7, (1988)

:104. K. Riedel, H. Eberhagen, 'O. Gruber et al., Nuclear Fusion 28, 1509 (1988).

105 . T.D. Rognlien, Conversion of the Bounce-Averaged Folkker-Planck Code to Conservation Form. Lawrence Livermor. National Laboratory; Livermore, CA, LCID-20474 (1985).

106. T.D. Rognlien, Phys. Fluids 26, 1545 (1983).

107. T.D. Rogrien and T.A. Brengle, Phys. Fluids 24, 871 (1981).

108. T.D. Rogrlien and T.A. Cutler, Nuclear Fusion 20, 1003 (1980).

:J09. S.J. Sackett, Calculation of Electromagnetic Fields and Forces in Coil Systems of Arbitrary Geametry. proc. 6th Symp. on Engineering Problems of Fusion Research (San Diego, Ca. 1975), IiLEE

110 S.J. Sackett, EFFI-A Code for Calculaling the Electromagnetic Field, Force. and Inductance in Coil Systems of A rbitrary Geometry, Lawrence Livermore National Laboratory, Livermore, CA: I:CRL-524U2 (1978).

111. H. St John. et al., Plasma Profile Analysis in DIII-D, 29 th Annual dpp meeting: Nov. 1987 (copies are available from the author).

112 A.G. Sgro. A. A. Mirin, and G. Marklin, Phys. Fluids 30, 3219 (1987).

113 C.E. Singer. Comm. Pl. Phys. and Cont. Fus. 11, 165 (1988).

114 C.E. Singer. D.E. Post, D.R. Wikkelsen, M.H. Kedi, A. Mckenney, A. Silverman, F.G.P. Seidl. P.H. Rutherford. R.J. Hawryluk. W.D. Langer, L. Foote, D.B. Heifetz, W. Houlberg, M.H. Hughes, R.Y. Jensen. G. Lister, and J. Ogden, "B.ALDCR: A One-Dimensional Plasma Transport Code," Computer Physics Communication 49, 275-398 (1988) (North-Holland, Amsterdam, CPCO(742).

115. G. Smith, "Alfvén Ion-Cyclotron Instability in Tandem-llirror Plasmas. 1," Phys. Flunds 27. 1499 (1984).

116. G. Smith, H. Berk, and L. LoDestro, "Compatibility of Alfvén Ion-Cyclotron and Magnetohydrodynamic Stability Criteria for an Axisymmetric Magnetic Mirror," Phys. Fluts 29 . 798 (1986). 


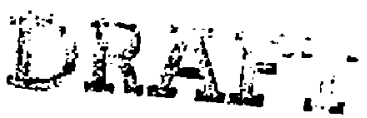

117. G. Smith. W. Nevins, and W. Sharp, "Alfven Ion-Cyclotron Instability in Tandem-Mirror Plasmas. 11," Phys. Fluids 27, 2120 (1984).

[118. T. Strait and R. Stambaugh, REVIEW-An Enhanced Alternative to Ploter, available in file doc:review.doc on the GA VAX cluster, (1986).

119, D. Strickler, Equilibrium Modeling of the TFCX Poloidal Field Coil System, ORNL/FEDC83/10. Sec 2.2

120. K. Tani, M. Suzuki, S. Yamomoto, M. Azumi, On the Analysis of Beam-Driven Current in a Tokamak, JAERI-M 88-042 (1988).

121: S. Tamor, "A Simple Fast Routine for Computation of Energy Transport by Synchrotron Radiaticn in Tokamaks and Similar Geometries," Science Applications, Inc. Report LAPS-72 S.A1-023081-189-LJ (1981), La Jolla, California.

122. S. Tamor, "Studies of Emission and Transport of Synchrotron Radiation in Tokamaks," Scjence Applications, Inc. Report LAPS-7I SAI-023-81-110LJ (1981), La Jolla, California.

123 C. Tull, LIBRISCODES, available on-line on the NMFECC Cray by typing document view libriscodes $/ \mathrm{t} \mathrm{v}$.

124'. E. Lchimoto, Ph.D. Thesis, Dept of Physics, Unir of Wisconsin-Madison (1988).

125' R.E. Wail z, R.R. Dominguez, and F.W. Perkins, GA-A19103 (Feb. 1988) to be published in Nuclear Fusion.

126. LCB-ERL technical memos

127. R.E. Walcz, Phys. Fluids 26, 3338 (1983)

128. R.E. Haltz, Fhys. Fluids 28, ธ̄77 (1985).

129. R.E. Walt2. Phys. Fluids 29, 3684 (1986)

130 R.E. Walt2. Phys. Fluids 31, 1962 (1988).

131 R.E. Waltz, Phys. Rev. Lett 55, 1098 (1985).

132 K.E. Waltz. R.R. Dominguez, and F.W. Perkins, G.A-A19103 (FEB. 1988) to be published in . Vuclear Fusion.

133 H. Wejtzer. CPAMI, pp. 929-932 (1985).

134 H. Mejtzner, K. Imre, D.B. Batchelor, Wave Propagatzon at lon Cyclotron Resunance $\mathrm{m}$ Large Toroids in Applicalions of Radio-Frequency Power to Plasmas AIP Conf. Proc. No 10\%, ed. S. Bernabei. R.H. Motley, Kissimmee, FL 1987.

155 KA. Werley, "One-and-a-Quarter-Dimensional Transport Modeling of the Field-Reversed Configuration." Phys. Fluids 30, 2119 (1987).

136. H.C. White, W.L. Bars, and R.W. Moir. DART: A Simulation Code for Charged Partıcle Beams, Lawrence Ljvermore National Laboratory, Li vermore, CA, LCID-21330 (1988).

137. P.A. Willmann, GRAFL-IIS: I'ser Oriented Sczence Gmphics, M-208, 24 (Dec. 198T). 
Index

ACCOME, 18

$A D, 19$

ALADDIN, 8

ALFWVS, 56

ambipolar potential. 24, 28. 50. 51

ANTENA 42

B2. 38

balance

particle. 16

power. 16

B.ALDLR, 29

BASIS, 6

BAV BACON 50

BEAM, 46

BETA, 20

BETA MC, 29

CART, 55

CFKX, 30

CHAPO, 30

CHIREG, 12

CQL. 5I

CQI.3D. 53

current drive

berst st rap, 18. 3.5

ech. 16. 25. 33. 41

lower hubrid. 16. 19

neutral beam. 16. 18. 23. 33, 35, 5r, 53

CYTRAN. $4 R$

DAHT. 25

data analysis. 12.15

DIII-D. 15

Fourier. 15

5t at istical. 12. 15

time series. 15

DATAPLOT, 12

DEG.A5, 4F:

DLi, $5 T$

DIPSI, 25

disruption thermal effects, 17

DRIFT. 58

edge physirs, 24.3B
EFFI, 11

EFIT, 20

electrode design, 25

EMONE, 25

ENERGY 15

EPFC, 20

EQPD, 21

ES1, 26

FE, YAT, 47

FIRC, 43

FLOKA, 34

Fokker-Planck, 50-53

multi-region. 52

multispecies. $\overline{1} 1$

FPT, 53

GARFIELD, 45

GATO, 54

GFLN3D, 11

GRAFL-III, 7

Graphics, 7, 8

heating

alpha, 16, 3i

ech. 24. 25.28 .33 .41 .50 .52 .53

ich, $24,25,28 ; 42,45,511$

neutral beam. $23,28,33,50-53$

ohmic. 16. 33. 37

HEQ. 21

HIB.G, 55

HORAC' $E, 48$

HPFB, 3]

HYBRID-I], 51

ICRH. 42

IDL. 13

IHT.IDC. 31

IIHRC, 14

LOCLS. 14

LTE, 18

YAFCO, 12

magnetic field equibrium DIII-D, 20 
open geometry, 18

torojdal geometry, 18, 20, 21, 32

permeable material, 11

vacuum, 11,12

MCGO, 23

MCNP, 10

MCPAT, 24

MFIRC, 43

MICE, 57

MINERYA, 32

MIRTH, 32

MIST, 40

MPPL, 7

MCMAK, 16

XEQ, 21

NFREYA, 45

ONETWO. 32

OPAKE2. 49

particle trajectory, 23,25

PDW1, 26

PDW2, 27

PDWZ, 2T

PELLET: 47

PEST. 54

PHLOW, 38

PLANET. 38

plasma absorption

electron crclutron, 41, 48, 49

plasma simulation. 25, 28

POPCON, 17

PROFREG, 14

Programming Language, $i$

radiation

bremst rahlung, 37

electron crclotron, 16. 41. 48, 49

impurity. 16. $3 i$

REVIEW, I6

If fields

electron cyclotron frequency 11

ion cyclotron frequency, 42,45

RFPBLRN. 33

RPD, 56

SFP. 51

SHIRC, 43
SIG, 15

SIGV, 9

simulation, 25

SMOKE, 52

S.AP, 34

stability

aic, 56

Alfvén, 51

mhd

open geometry, 18, 54

toroidal geometry, 54

parametric, 25,28

stellerator: 36

TADDPACK, 17

TEARING, $\mathbf{5 4}$

TEBASCO, 18

TESS, 28

TETAA, 17

THEORY', 35

THIRC, 44

TIBRO. 23

tokamak design, 16, 17

TORA\}, 41

TORCH, 41

TRACK, 34

TRANSP, 35

transport

axial. 24.38

cross-field. 29. 31). 35. 38. 53

impurity, 40

multispecies, 29

neutra particle, $29,46,47$

neutron, 10

photon, 10

velocity space, 59.52

TSC. 36

THOFLD. 58

$1 E Q, 21$

VXEC: 21

l.MOMS. 22

I'S], $28^{\prime}$

WHIST, 36

WPCP38, 49

XPOSTPLT, \& 
ن

ZCODE, 39

ZERO. 37

ZOHAR, 28 